ARTICLE

\title{
Long non-coding RNA RAMS11 promotes metastatic colorectal cancer progression
}

Jessica M. Silva-Fisher (1) 1,2, Ha X. Dang ${ }^{1,2,3}$, Nicole M. White ${ }^{1,2}$, Matthew S. Strand ${ }^{4}$, Bradley A. Krasnick ${ }^{4}$, Emily B. Rozycki ${ }^{1}$, Gejae G. L. Jeffers ${ }^{1}$, Julie G. Grossman (1) 4, Maureen K. Highkin ${ }^{1}$, Cynthia Tang (1) 1, Christopher R. Cabanski ${ }^{5}$, Abdallah Eteleeb1, Jacqueline Mudd ${ }^{4}$, S. Peter Goedegebuure ${ }^{4}$, Jingqin Luo ${ }^{2,6}$, Elaine R. Mardis (1) 7, Richard K. Wilson7, Timothy J. Ley (1) ${ }^{1,2}$, Albert C. Lockhart ${ }^{8}$, Ryan C. Fields ${ }^{2,4,10} \&$ Christopher A. Maher (1,2,3,9,10凶

Colorectal cancer (CRC) is the most common gastrointestinal malignancy in the U.S.A. and approximately $50 \%$ of patients develop metastatic disease (mCRC). Despite our understanding of long non-coding RNAs (IncRNAs) in primary colon cancer, their role in $\mathrm{mCRC}$ and treatment resistance remains poorly characterized. Therefore, through transcriptome sequencing of normal, primary, and distant mCRC tissues we find 148 differentially expressed RNAs Associated with Metastasis (RAMS). We prioritize RAMS11 due to its association with poor diseasefree survival and promotion of aggressive phenotypes in vitro and in vivo. A FDA-approved drug high-throughput viability assay shows that elevated RAMS11 expression increases resistance to topoisomerase inhibitors. Subsequent experiments demonstrate RAMS11-dependent recruitment of Chromobox protein 4 (CBX4) transcriptionally activates Topoisomerase II alpha (TOP2 $\alpha$ ). Overall, recent clinical trials using topoisomerase inhibitors coupled with our findings of RAMS11-dependent regulation of TOP2 $\alpha$ supports the potential use of RAMS11 as a biomarker and therapeutic target for mCRC.

\footnotetext{
${ }^{1}$ Department of Internal Medicine, Washington University School of Medicine, St. Louis, MO, USA. ${ }^{2}$ Alvin J. Siteman Cancer Center, Washington University School of Medicine, St. Louis, MO, USA. ${ }^{3}$ The McDonnell Genome Institute, St. Louis, MO, USA. ${ }^{4}$ Department of Surgery, Washington University School of Medicine, St. Louis, MO, USA. ${ }^{5}$ Parker Institute for Cancer Immunotherapy, San Francisco, CA, USA. ${ }^{6}$ Division of Public Health Sciences, Department of Surgery, Washington University School of Medicine, St. Louis, MO, USA. 7 Institute for Genomic Medicine, Nationwide Children's Hospital, Columbus, OH, USA. ${ }^{8}$ Department of Medicine, University of Miami, Miami, FL, USA. ${ }^{9}$ Department of Biomedical Engineering, Washington University School of Medicine, St. Louis, MO, USA. ${ }^{10}$ These authors contributed equally: Ryan C. Fields, Christopher A. Maher. ${ }^{凶}$ email: christophermaher@wustl.edu
} 
C olorectal cancer (CRC) is the most common gastrointestinal malignancy in the United States. At the time of initial diagnosis, $20 \%$ of patients present with metastasis, and of those patients with primary disease approximately $50 \%$ will eventually develop metastatic disease ${ }^{1}$. Furthermore, the overall 5-year survival rate for patients with metastatic CRC (mCRC) is only $14 \%^{2,3}$. Currently, there are numerous therapeutic treatments for patients with mCRC including surgery, cytotoxic chemotherapy, targeted therapy, immunotherapy, radiation, and combination strategies. However, there is little pretreatment data that can predict response to treatment and development of resistance ${ }^{4}$. While there are promising developments in second-line treatment options for mCRC patients using cytotoxic agents or targeted agents, the mechanisms driving metastatic progression remain poorly characterized thus prohibiting effective drug development. Furthermore, response to second-line treatment is even less effective than first line ${ }^{5,6}$. These statistics and poor treatment options highlight the critical need for improved biomarker-driven therapies at the time of diagnosis.

To date, CRC research has primarily focused on the deregulation of protein-coding genes to identify oncogenes and tumor suppressors as potential diagnostic and therapeutic targets ${ }^{7,8}$. While more recent studies have explored the role of microRNAs in $\mathrm{CRC}^{9.10}$, there is still a lack of studies focusing on long noncoding RNAs (lncRNAs) in mCRC. LncRNAs are typically greater than 200 nucleotides in length, lack coding potential, are transcribed by RNA polymerase II, spliced, $5^{\prime}$ capped, and polyadenylated ${ }^{11}$. LncRNAs are known to have a diverse range of biological functions, including serving as critical regulators in tumorigenesis and metastasis ${ }^{12-18}$. Furthermore, the clinical significance of lncRNAs can be exemplified by their use as diagnostic, prognostic, predictive biomarkers, and potential therapeutic targets ${ }^{19-24}$. Therefore, the characterization of lncRNAs, elucidating their function, and assessing their clinical applicability could significantly impact mCRC diagnosis and treatment.

While transcriptome sequencing has provided an unbiased method for discovering lncRNAs, existing large-scale sequencing projects such as The Cancer Genome Atlas Network (TCGA) ${ }^{25}$ are comprised of predominantly primary tumors lacking matched metastatic samples. This represents a critical barrier to discovering novel lncRNAs throughout the progression of primary to metastatic disease correlated to treatment response and resistance. To address this, we have conducted a meta-analysis of normal, primary, and distant metastatic tissues from CRC patients across two independent patient cohorts to discover differentially expressed (DE) lncRNAs in metastatic tumors compared with primary tumors, termed RNAs Associated with Metastasis $(R A M S)$. We have prioritized RAMS11 as it was a top up-regulated lncRNA in metastasis and associated with poor disease-free survival across multiple cohorts. We then demonstrate that RAMS11 promotes aggressive phenotypes in vitro and in vivo. While lncRNAs have been shown to promote tumor progression $^{26-28}$, the understanding of their role in treatment resistance is still unknown. Therefore, we have utilized a drug screen to discover that RAMS11 promotes resistance to topoisomerase inhibitors and provide mechanistic insight into RAMS11-dependent topoisomerase II alpha (TOP2 $\alpha$ ) regulation to promote $\mathrm{mCRC}$.

\section{Results}

LncRNA landscape of mCRC. To identify consistently altered lncRNAs during mCRC, we performed transcriptome sequencing and analysis of 37 patients from two independent cohorts. The first cohort includes ten normal colon epithelium, two primary
CRC, and fourteen distant mCRC patient samples collected from Washington University, termed WUSTL cohort. The second cohort is from a previously published transcriptome sequencing study by Kim et al. ${ }^{29}$ using matched normal, primary, and metastatic samples from 18 CRC patients, termed Kim cohort (Fig. 1a).

To identify lncRNAs altered in the metastatic samples relative to primary and normal samples, we performed a meta-analysis of the WUSTL and Kim cohorts. We identified 148 DE lncRNAs (FDR < 0.05 , fold change $>2$ ) in metastasis, termed RAMS (Fig. $1 \mathrm{~b}$ and Supplementary Data 1). Several previously well-known and characterized lncRNAs known to promote oncogenic phenotypes in CRC or other cancer types were also detected. This includes increased expression of H19, HULC, CCAT4, and TCONS_I2_ 00022545 in $\mathrm{mCRC}$ and decreased expression of FENDRR in metastatic samples ${ }^{30-34}$ (Fig. 1b). Overall, this serves as a key meta-analysis from aggressive CRC patient tissues to establish the mCRC lncRNA landscape.

RAMS11 is upregulated in MCRC. We prioritized our functional studies on lncRNAs that were highly deregulated and potentially clinically relevant in mCRC. To prioritize all RAMS, we evaluated whether their expression correlated with patient outcome. First, we found that six of the 148 RAMS were associated with diseasefree survival using 232 patients from the TCGA CRC cohort (RNA-Seq). Among the six RAMS associated with survival in the TCGA cohort, only RAMS11 was associated with poor survival from a second cohort of 82 patients (Fig. 1a, c) from the Sveen study (GSE24549, exon array ${ }^{35}$ ). These results indicate that high levels of RAMS11 in primary tumors may serve as an indication of poor patient outcome. Notably, RAMS11 was also a top upregulated lncRNA in metastatic tumors $(\mathrm{FPKM}=4.81)$ as compared with primary tumors (combined $p=2.56 \times 10^{-10}$ average fold change $=6.1$ ) and normal tissues (combined $p=$ $2.2 \times 10^{-20}$, average fold change $=12.9$ ) (Fig. 1d and Supplementary Fig. 1a). We further validated the upregulation of RAMS11 by qPCR when comparing matched metastatic patient samples with normal ( $p=0.007$, two-tailed paired $t$-test) and primary $(p=0.024$, two-tailed paired $t$-test) patient samples (Supplementary Fig. 1b).

Our de novo transcript assembly using the WUSTL cohort identified RAMS11 as a five-exon transcript of 959 nucleotides, which we confirmed by $5^{\prime}$ and $3^{\prime}$ rapid amplification of cDNA ends (RACE) (Fig. 1d, Supplementary Data 2). Previously, three exons of the RAMS11 transcript were annotated as LINC01564 (NR_125841) through a microarray probe-based analysis ${ }^{36}$ (Fig. 1d). We further characterized RAMS11 expression in a panel of CRC cell lines. RAMS11 was highly expressed in a panel of six primary (more than three-fold increase) and two mCRC cell lines (more than 11-fold increase) compared with CCD18-Co, a normal colon control cell line (Supplementary Fig. 1c). Since the cellular localization of lncRNAs can help decipher their functions, we fractionated LoVo mCRC cells with high endogenous expression of RAMS11. As shown in Supplementary Fig. 1d, RAMS11 is predominantly expressed in the nucleus (89.5\%), with only a $10.5 \%$ expression in the cytoplasm. Taken together, these results show that RAMS11 is a five-exon, nuclear localized, lncRNA that is highly expressed in primary and mCRC cell lines and absent in normal colon epithelium.

RAMS11 promotes aggressive phenotypes in vitro. To understand RAMS11 functional significance, we created a RAMS11 knockout (KO) model by generating two CRISPR/Cas9 luciferasetagged cell lines with a genomic deletion of the last four exons of RAMS11 in the LoVo metastatic colon cancer cell line 
a

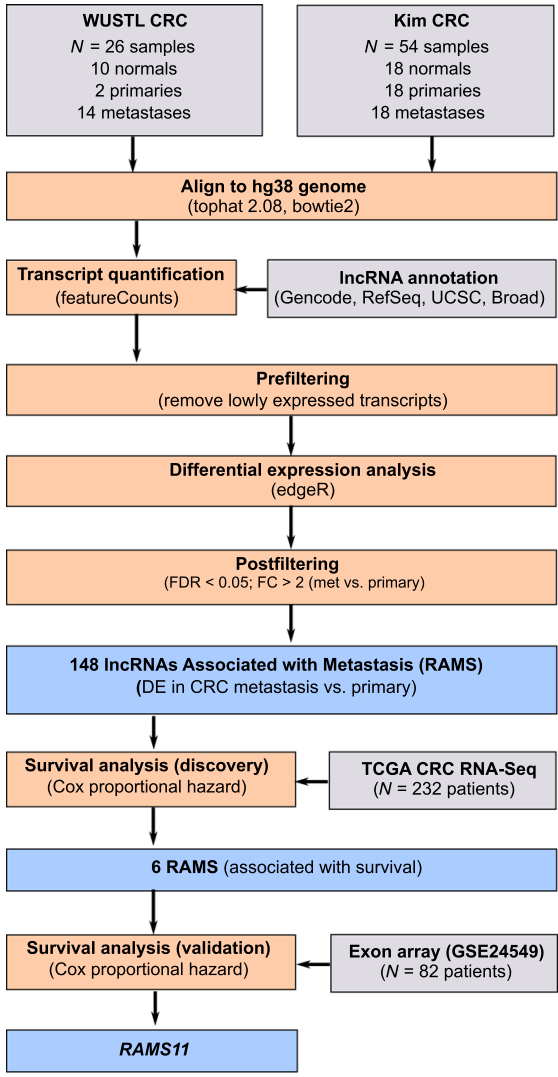

b

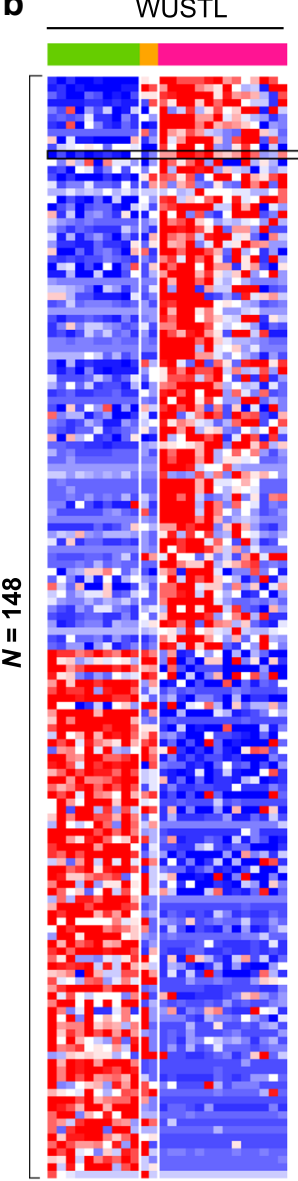

$\square$ Normal

$\square$ Primary

$\square$ Liver metastasis
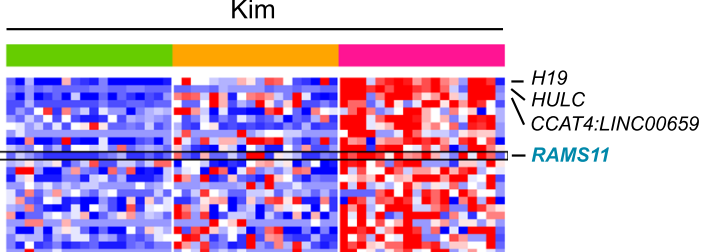

Trentring

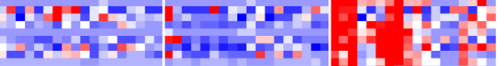

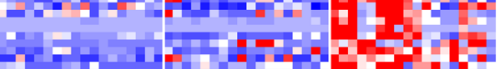
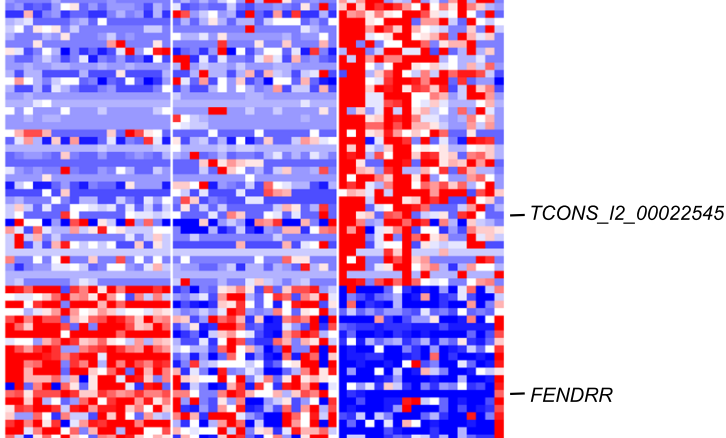

.
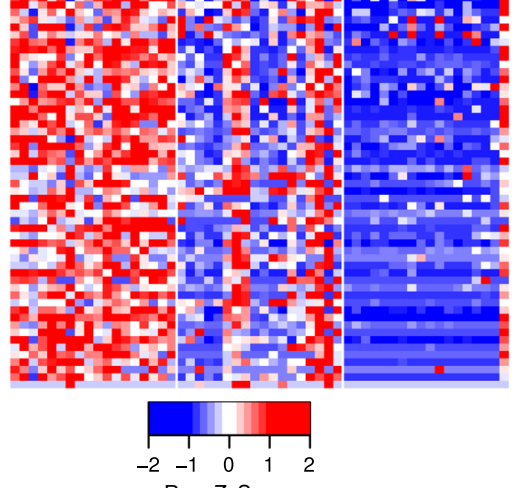

Row Z-Score
C

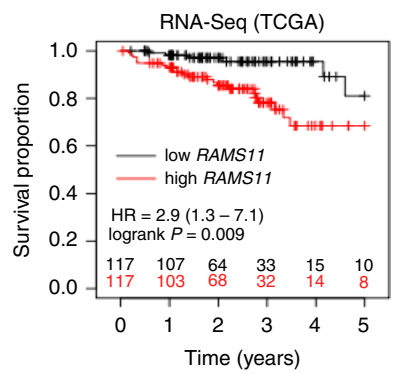

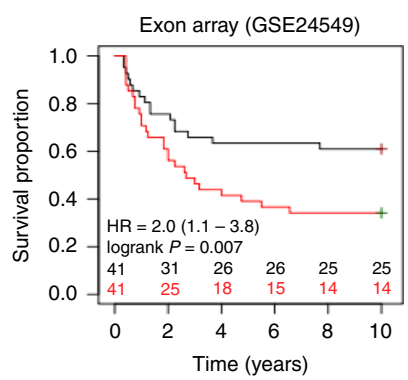
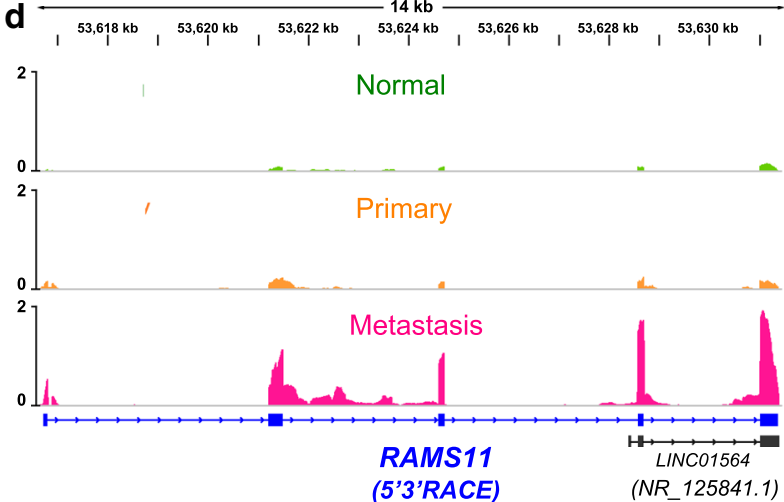

Fig. 1 RNAs associated with metastasis (RAMS). a Analysis pipeline for discovery of metastatic CRC IncRNAs. Shaded gray color boxes (input), orange color boxes (analysis), and blue color boxes (output/results). b Heatmap of IncRNAs differentially expressed in metastasis compared with primary. Patient samples are indicated on top row shown as normal (green), primary (orange), and liver metastasis (pink). Heatmap color is scaled by row expression Zscore. c Kaplan-Meir plots showing RAMS11 association with poor disease-free survival in The Cancer Genome Atlas (TCGA) RNA-Seq and exon array (GSE24549) datasets. Numbers above $x$-axis are patients at risk at the intervals. $p$ values are inferred from a two-sided logrank test. $\mathbf{d}$ Average normalized RNA-Seq coverage across WUSTL and Kim cohorts. Normal samples are green boxes, primary samples are orange boxes, and metastatic samples are pink boxes. 5'3' RACE validated five-exon sequence is shown below in blue.

(Supplementary Fig. 2a). We confirmed greater than a $99.9 \%$ reduction in our RAMS11 CRISPR KO models (clones referred to as CRISPR1 and CRISPR2) relative to wild-type cells (Fig. 2a) and confirmed that the genomic deletion of RAMS11 did not alter the expression of adjacent genes GCLC and KLH31 (Supplementary Fig. 2b, c).
We used these genetically engineered cell lines to determine changes in the invasiveness of cells using Matrigel-coated transwells in a modified Boyden chamber assay. There was more than a $60 \%$ decrease in invasion of RAMS11 CRISPR KO cells (CRISPR1 $p=0.004$, CRISPR2 $p=0.023$, two-tailed paired $t$-test) compared with wild-type cells (Fig. $2 \mathrm{c}$, e). We also conducted a transient 
a

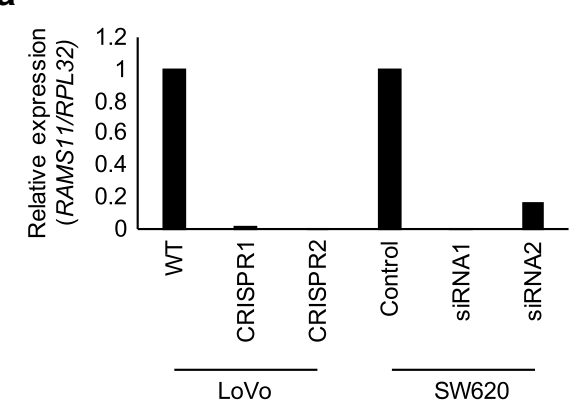

b

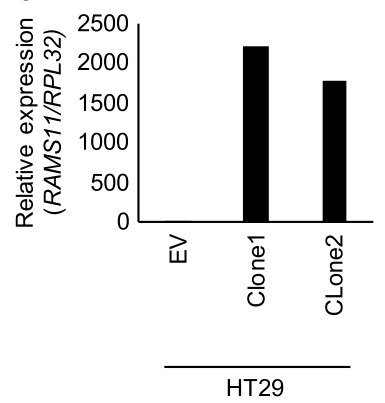

c

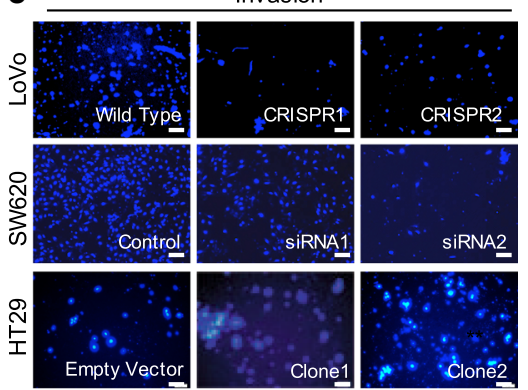

d

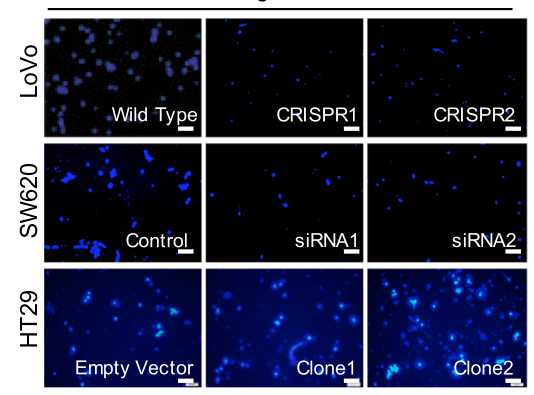

h

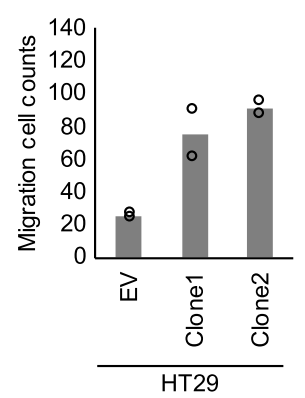

e

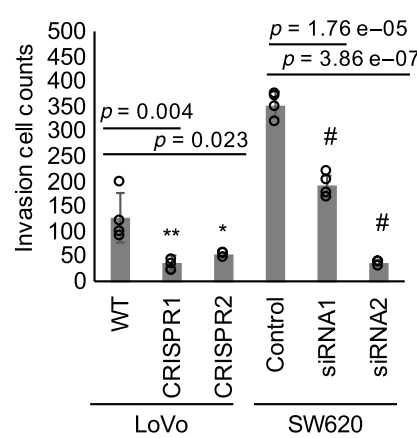

f

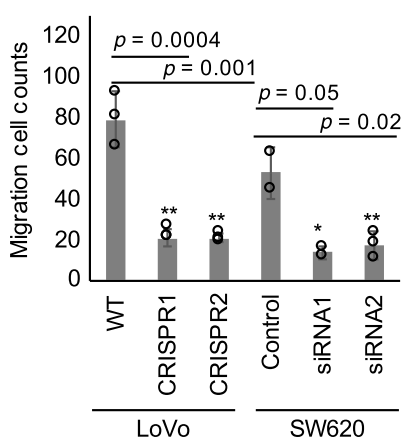

g

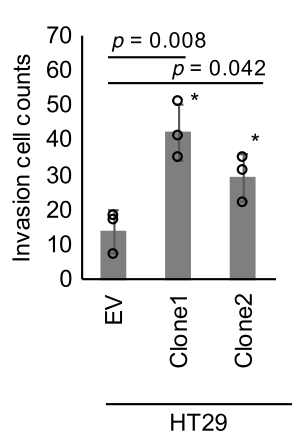

i

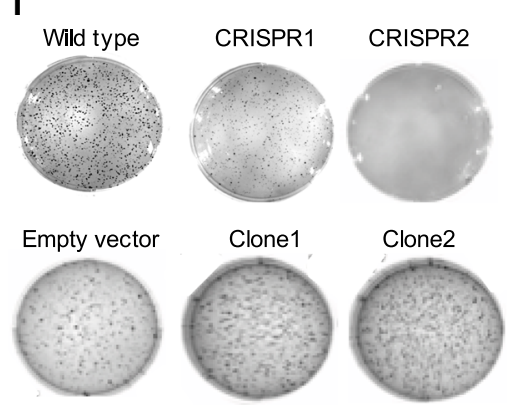

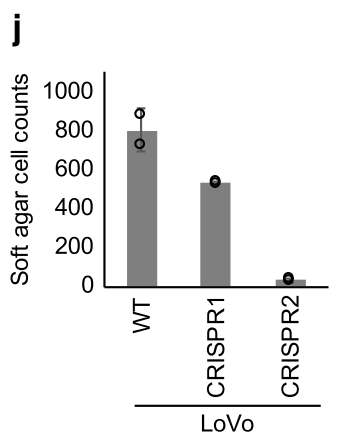

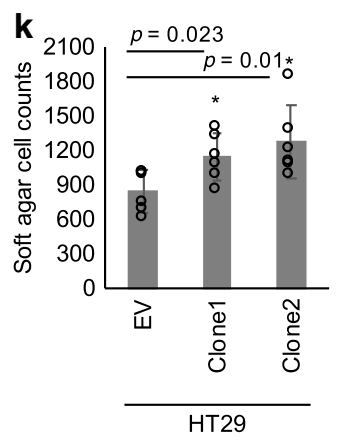

Fig. 2 RAMS11 promotes an invasive phenotype. Expression of RAMS11 in a LoVo CRISPR KO, SW620 silenced cells, and b HT29 overexpressing cells (Clone1 and Clone2) as measured by qPCR. c Images of DAPI-stained LoVo RAMS11 CRISPR KO cells and SW620 siRNA silenced cells show decreased invasion compared with controls. HT29 cell lines overexpressing RAMS11 show increased invasion. d Images of LoVo RAMS11 CRISPR KO cell lines and SW620 cells transfected with RAMS11 siRNAs show decreased migration. HT29 cell lines overexpressing RAMS11 show increased migration. e, $\mathbf{f}$ Quantification of invaded $(n=4)$ and migrated cells $(n=3)$ in LoVo and SW620 cells. $\mathbf{g}, \mathbf{h}$ Quantification of invaded $(n=3)$ and migrated cells $(n=2)$ in HT29 cells. i RAMS11 CRISPR KO cells decreased growth on soft agar $(n=2)$ and RAMS11 overexpressing cells $(n=6)$ increased growth on soft agar. $\mathbf{j}, \mathbf{k}$ Quantification of soft agar cells. All data are presented as mean values \pm s.d, analyzed by two-tailed paired $t$-test, and repeated more than two times. Bar $=25 \mu M,{ }^{\star} p<0.05,{ }^{\star \star} p<0.005$, $\#_{p}<0.0005$. Source data are provided as a Source Data File.

knockdown of RAMS11 in a second colon cancer metastatic cell line, SW620, and observed at least $80 \%$ knockdown in two independent siRNAs (Fig. 2a). We saw a 50\% decrease of invaded cells relative to control cells that were transfected with scrambled siRNA $(p<0.00005$, two-tailed paired $t$-test, Fig. $2 c$, e). Conversely, stably overexpressing RAMS11 (Clone1 and Clone2) in HT29 cells, with low endogenous RAMS11 expression (Fig. 2b), resulted in a 53\% increase in cellular invasion (Clone1 $p=0.008$, Clone2 $p=0.042$, two-tailed paired $t$-test) relative to the empty vector control cell line (Fig. 2c, g). Further, we rescued the number of invaded cells to wild-type levels with transient overexpression of RAMS11. Our CRISPR KO models re-expressing RAMS11 (CRISPR RAMS11 OE) revealed more than a $60 \%$ increase of invaded cells relative to the CRISPR KO cell lines (CRISPR1 RAMS11 OE and CRISPR2 RAMS11 OE $p<$ 0.00005 , two-tailed paired $t$-test) (Supplementary Fig. $2 \mathrm{~d}$, e). We also observed a $73 \%$ decrease in cellular migration in the
CRISPR KO cells (CRISPR1 and CRISPR2 $p<0.0005$, two-tailed paired $t$-test) and more than $67 \%$ decrease in SW620 RAMS11 silenced cells $(p<0.05$, two-tailed paired $t$-test) (Fig. $2 \mathrm{~d}, \mathrm{f})$. In addition, there was increased migration in HT29 RAMS11 overexpressing cells (Fig. 2d, h). Taken together, this demonstrates that RAMS11 promotes cellular invasion in CRC.

We next investigated the ability of RAMS11 to promote anchorage-independent growth as another indication of aggressive oncogenic phenotypes. Using a soft agar colony formation assay, we detected more than a $66 \%$ reduction in colony formation in the RAMS11 CRISPR KO cell lines relative to the wild-type LoVo cell line (Fig. 2i, j). Conversely, there was a 30\% increase in colony formation in the RAMS11 overexpressing HT29 cell lines (Clone1 and Clone $2 p<0.05$, two-tailed paired $t$-test) compared with the empty vector cell line (Fig. $2 \mathrm{i}, \mathrm{k}$ ). These combined data show that decreased expression of RAMS11 in genetically modified and transient knockdown cell lines mitigates 
aggressive phenotypes, while overexpressing RAMS11 promotes aggressive phenotypes.

As another hallmark of aggressive phenotypes, we next assessed the effect of RAMS11 expression on cellular proliferation. We observed a $27 \%$ decrease in proliferation in our CRISPR KO cell lines $(p<0.05$, two-tailed paired $t$-test) (Supplementary Figs. $2 \mathrm{f}$, g, and 10). Taken together, our in vitro data demonstrate that RAMS11 can promote multiple oncogenic phenotypes.

Next, we evaluated whether RAMS11 is broadly deregulated across cancer types, which would suggest a critical conserved oncogenic role in cancer progression, which we refer to as an oncolncRNA ${ }^{37}$. We conducted a pan-cancer analysis of 6984 tissues comprised of matched and unmatched normal and primary tumors across 22 different cancer types studied within the TCGA. This analysis revealed that RAMS11 had elevated expression in primary tumors compared with normal tissue of origin in colorectal adenocarcinoma $(p<0.00001)$ and four additional cancer types including: lung adenocarcinoma $(p<0.00001)$, lung squamous cell carcinoma $(p<0.00001)$, head and neck squamous cell carcinoma $(p<0.00001)$, and kidney renal papillary cell carcinoma $(p<0.00001)$ (Supplementary Fig. 3a).

Last, since we found that RAMS11 is an onco-lncRNA upregulated across cancer types, we determined if RAMS11 also promoted oncogenic phenotypes in additional cancer types. Therefore, we silenced RAMS11 expression and assessed invasion in two different histologies of non-small cell lung cancer, lung squamous (HCC95) and lung adenocarcinoma (A549), cell line models. We found that silencing RAMS11 expression in both cancer cell lines caused a decrease in cellular invasion (HCC95 $p<0.05$; A549 $p<0.005$, two-tailed paired $t$-test) (Supplementary Fig. $3 \mathrm{~b}-\mathrm{g}$ ). These results indicate that increased RAMS11 expression promotes oncogenic phenotypes in multiple cancer types.

RAMS11 promotes tumor growth and metastasis in vivo. Since our RAMS11 CRISPR KO lines had a significant decrease in cellular proliferation in vitro (Supplementary Fig. $2 \mathrm{f}, \mathrm{g}$ ), we evaluated tumor growth by injecting RAMS11 CRISPR KO cells into NOD/SCID immunocompromised mice. Twenty-five days after subcutaneous injection of LoVo luciferase-tagged wild-type and our luciferase-tagged RAMS11 CRISPR KO cells we found a significant decrease $(p<0.0005$, two-tailed paired $t$-test $)$ in both tumor volume and size in mice injected with RAMS11 CRISPR KO cells compared with wild-type cells (Fig. 3a-d). These results indicate that RAMS11 may indeed induce tumor formation and promote oncogenesis in vivo.

Next, to assess the contribution of RAMS11 to cause metastasis in vivo, we used two mouse models of metastasis: a tail vein injection model to study the development of lung metastases and a hemisplenectomy model to study the development of liver metastases. For the tail vein model, we injected LoVo luciferasetagged wild-type and our luciferase-tagged RAMS11 CRISPR KO cells into the tail vein of 5 -week-old NOD/SCID mice. We monitored the mice at day 0 , within 30 min to $1 \mathrm{~h}$ post injection, and weekly for metastasis formation with bioluminescence imaging (BLI). All mice were injected successfully showing similar luminescence levels determined by BLI at baseline day 0 (Fig. 4a). Further, images from day 7 showed no detectable signal indicating the internalization of circulating cells throughout the mouse. There was little or no lung metastasis in mice injected with RAMS11 CRISPR KO cell lines by day 35 ( $p=0.02$, twotailed paired $t$-test) as compared with wild-type cells (Fig. $4 \mathrm{a}, \mathrm{b}$ ). We continued to monitor lung metastasis for 91 days and saw significantly less lung metastasis in RAMS11 CRISPR KO cell-injected mice compared with wild-type cell-injected mice
( $p<0.05$, two-tailed paired $t$-test) (Fig. $4 \mathrm{~b}, \mathrm{c}$ ). We also detected less lung metastasis ex vivo in mice injected with RAMS11 CRISPR KO cells compared with mice injected with wild-type cells (Fig. 4d). In addition, extracted lungs had little to no tumors detected by hematoxylin and eosin (H\&E) stain and lower levels of Ki67 staining from RAMS11 CRISPR KO cell-injected mice compared with wild-type cell-injected mice (Fig. 4e).

We assessed if RAMS11 promoted liver metastasis using the hemisplenectomy model. LoVo luciferase-tagged wild-type and luciferase-tagged RAMS11 CRISPR KO cells were injected into 8 -week-old NGS mouse spleens ${ }^{38}$. We detected liver metastasis by day 7 in mice injected with wild-type cells (Fig. 5a) and detected significantly lower levels of bioluminescence in RAMS11 CRISPR KO cell-injected mice compared with wild-type cellinjected mice by Day 21 (CRISPR1 $p=0.016$, CRISPR2 $p=$ 0.008 , two-tailed paired $t$-test, Fig. $5 \mathrm{a}, \mathrm{b}$ ). We excised all mouse livers and validated the decrease of liver metastasis (Fig. 5c), decreased liver weights (CRISPR1 $p=0.0000026$, CRISPR2 $p=$ 0.00069 , two-tailed paired $t$-test, Fig. $5 \mathrm{~d}$ ), and decrease in overall liver metastasis area (CRISPR1 $p=0.00021$, CRISPR2 $p=$ 0.00018 , two-tailed paired $t$-test, Fig. $5 \mathrm{e}$ ) in RAMS11 CRISPR KO cell-injected tumors. Decreased tumor burden and proliferation in RAMS11 CRISPR KO cell livers were further determined by $\mathrm{H} \& \mathrm{E}$ and Ki67 staining (Fig. 5f). Overall, our cell models manipulating RAMS11 expression demonstrate the ability of RAMS11 to promote invasive phenotypes both in vitro and in vivo.

Drug screen reveals $R A M S 11$ resistance to TOP2 $\alpha$ inhibitors. To implicate RAMS11 in specific biological processes and establish its clinical importance, we conducted a high-throughput viability assay using 119 FDA-approved anticancer drugs from the NIH Developmental Therapeutics Program (Approved Oncology Drugs Set VI). The FDA-approved anticancer panel included multiple classes of drugs such as kinase inhibitors, alkylating agents, antineoplastic antibiotics, anthracycline antibiotics, and antineoplastic agents (topoisomerase inhibitors). The HT29 RAMS11 overexpressing and control cells were treated for $72 \mathrm{~h}$ to assess cellular viability upon drug treatment (Supplementary Fig. 4a and Supplementary Data 3). The RAMS11 overexpressing cells were resistant to nine drugs as demonstrated by a greater than three-fold increase in cellular viability when compared with the empty vector control cell line with the greatest resistance observed with gemcitabine and floxuridine (FUDR) (Supplementary Fig. 4b, c). FUDR, a 5-FU derivative, is commonly used to treat $\mathrm{mCRC}$, while gemcitabine is used in refractory mCRC ${ }^{39-42}$. Due to 5 -FU commonly used to treat mCRC, we further determined if RAMS11 expression altered drug sensitivity in treated cells. In our RAMS11 CRISPR KO lines we found a 1.7 -fold and 5.8-fold increase in drug sensitivity in CRISPR1 and CRISPR2, respectively, compared with wild-type cells (Supplementary Fig. 5a). Similarly in SW620 cells with transient silencing of RAMS11 there was a greater than 1.5-fold increase in drug sensitivity in both siRNAs (siRNA1 fold $>1.53$, siRNA2 fold $>1.59$ ) relative to scrambled control wild-type treated cells (Supplementary Fig. 5b). 5-FU, irinotecan (topoisomerase I inhibitor $(T O P 1)$ ), and oxaliplatin (new-generation platinum compound) are currently used as first-line active chemotherapy options individually or in combination for patients with metastatic disease $\mathrm{e}^{4,43,44}$. We did not see a significant effect of cell viability for irinotecan or oxaliplatin using our HT29 RAMS11 overexpressing cells or LoVo RAMS11 CRISPR KO cells (Supplementary Data 3, Supplementary Fig 5c-e). SW620 cells with silenced RAMS11 also did not have a significant effect of cellular viability for oxaliplatin treatment, but we did detect an increase in 


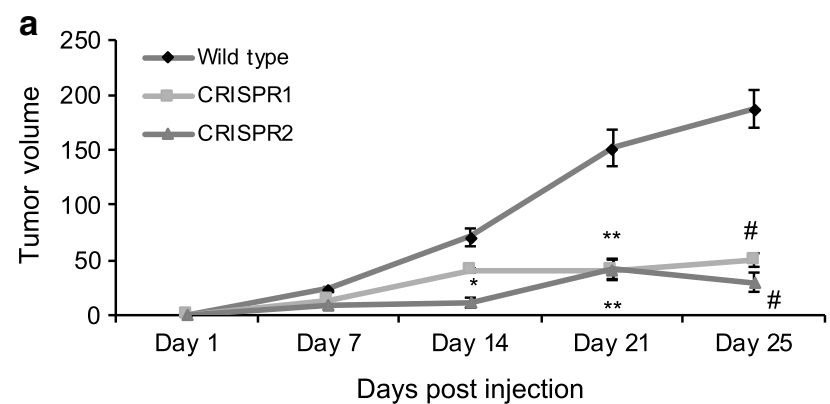

C

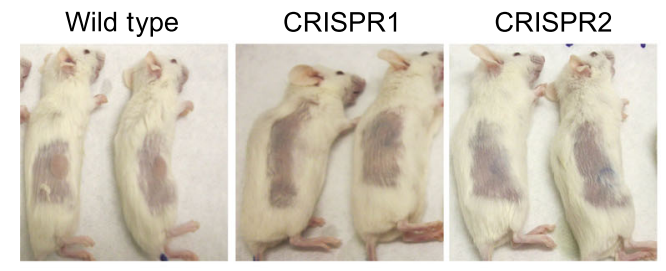

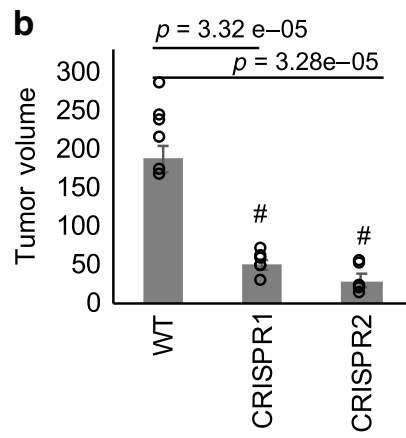

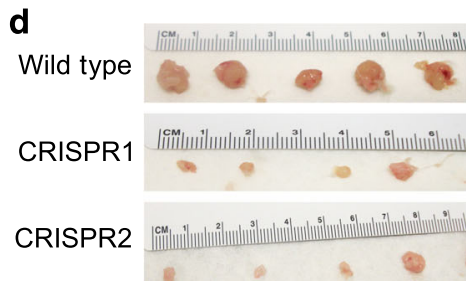

Fig. 3 RAMS11 induces tumor growth in vivo. a Significant decrease in tumor growth in RAMS11 CRISPR KO subcutaneous injected mice. Day 14 CRISPR1 $p=0.01$ CRISPR2 $p=0.0002$, day 21 CRISPR1 $p=0.00002$ CRISPR2 $p=0.0006$, day 25 CRISPR1 $p=3.32 \mathrm{e}-05$ CRISPR2 $p=3.28 \mathrm{e}-05$. b Quantification at day 25 showing decreased tumor growth in RAMS11 CRISPR KO lines compared with wild type. c Representative mice showing little to no tumor growth and $\mathbf{d}$ representative resected tumors from mice. Data shown as mean \pm SEM and analyzed by two-tailed paired $t$-test, with $n=10$ per group repeated two times. ${ }^{\star} p<0.05,{ }^{\star \star} p<0.005,{ }^{\#} p<0.0005$. Source data are provided as a Source Data File.

a

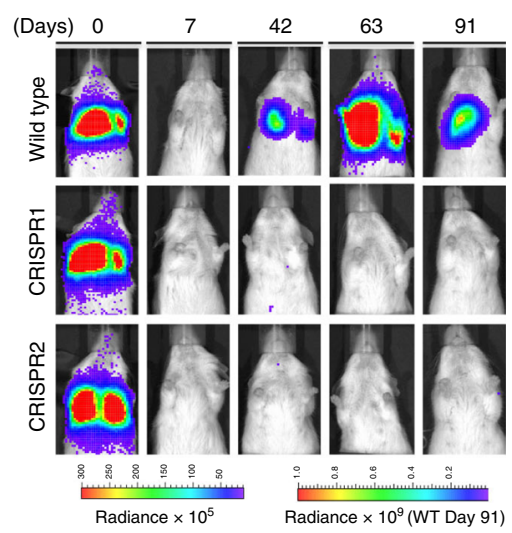

C

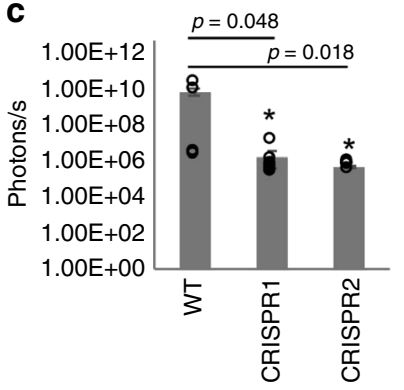

b

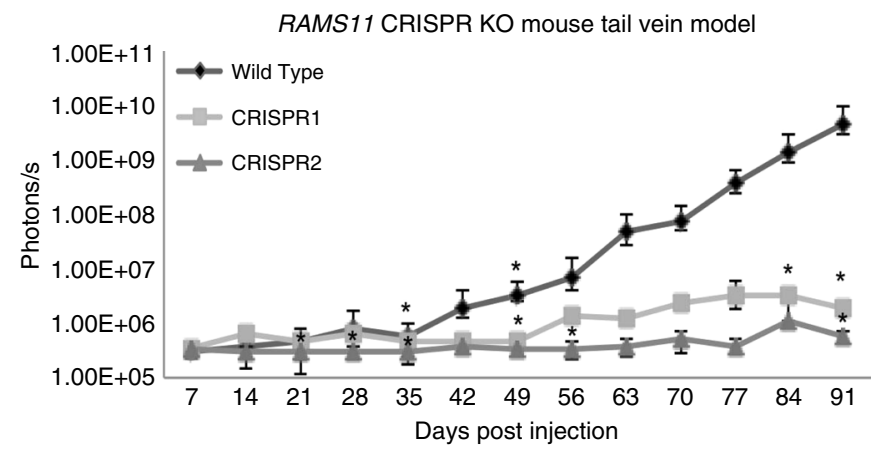

e

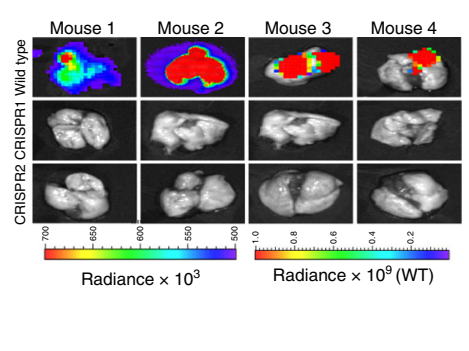

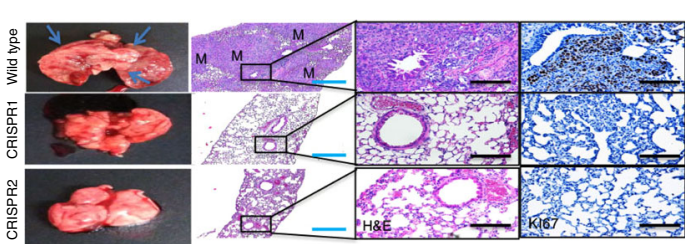

Fig. 4 RAMS11 induces lung metastasis via tail vein mouse model. a Representative mice and $\mathbf{b}$ quantification showing no lung metastasis in $R A M S 11$ CRISPR KO cell-injected mouse by BLI. Day 28 CRISPR2 $p=0.04$, day 35 CRISPR1 $p=0.02$ CRISPR2 $p=0.013$, day 49 CRISPR1 $p=0.02$ CRISPR2 $p=$ 0.01 day 84 CRISPR1 $p=0.05$ CRISPR2 $p=0.03$, day 91 CRISPR1 $p=0.04$ CRISPR2 $p=0.01$. c, d Day 91 ex vivo mouse lungs show RAMS11 CRISPR KO cell-injected mice have decreased lung metastasis by BLI. e Hematoxylin and eosin stain showing metastasis (M) and Ki67 stain. Three independent tissues were stained per group. Blue bar $=1 \mathrm{mM}$, black bar $=25 \mu \mathrm{M}$. Data shown as mean \pm SEM and analyzed by two-tailed paired $t$-test, with $n=12$ per group repeated two times. ${ }^{\star} p<0.05$. Source data are provided as a Source Data File.

drug sensitivity to irinotecan (siRNA1 fold $>3.17$ and siRNA2 fold $>11.8$, Supplementary Fig 5f).

Interestingly, half of the topoisomerase inhibitors assessed caused at least a two-fold increase in drug resistance in the HT29
RAMS11 overexpressing cells including the TOP1 topotecan hydrochloride $(\mathrm{HCl})$ and four TOP2 $\alpha$ inhibitors (doxorubicin $\mathrm{HCl}$, epirubicin $\mathrm{HCl}$, daunorubcin $\mathrm{HCl}$, and idarubicin $\mathrm{HCl}$ (Fig. 6a, b). To further support our observation that RAMS11 
a

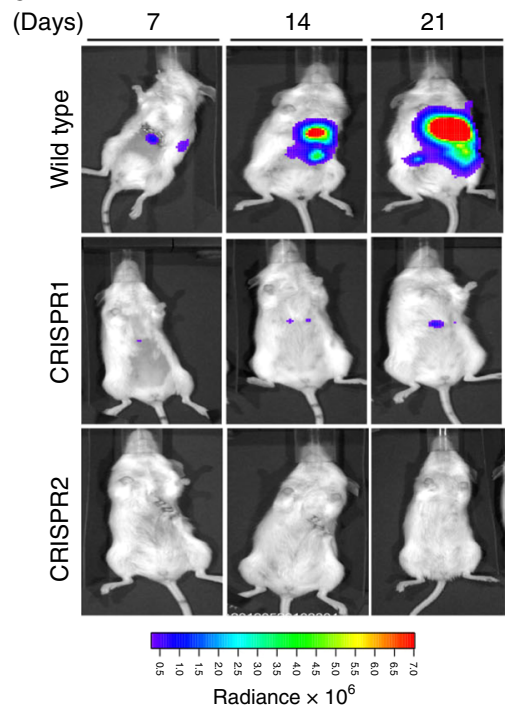

b

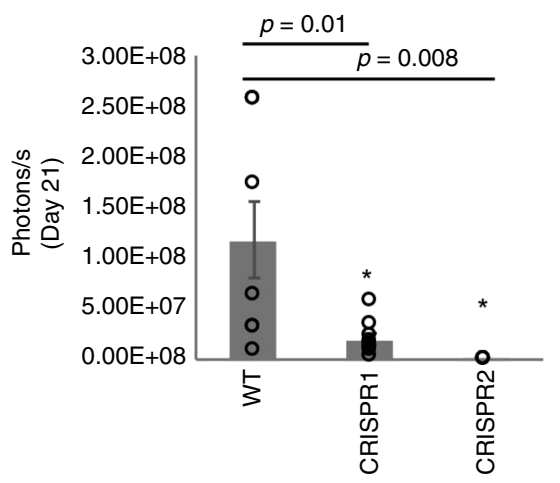

C

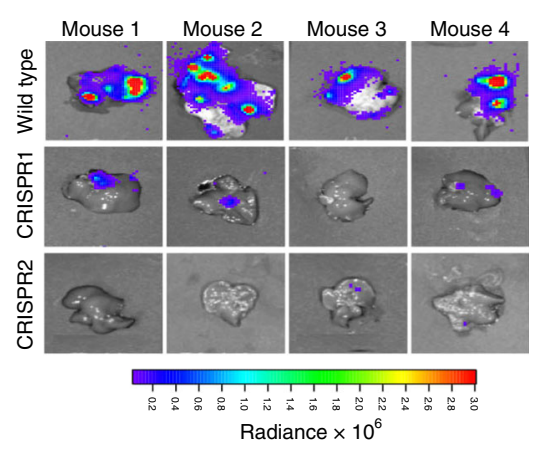

d

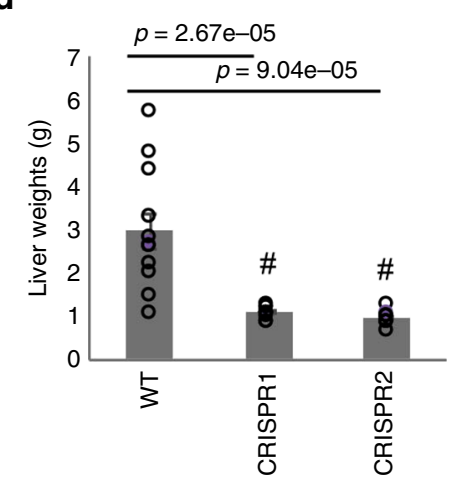

e

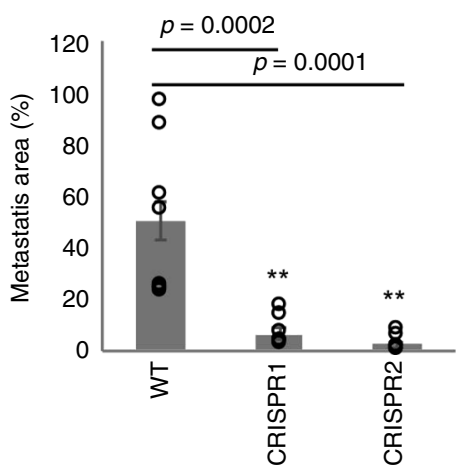

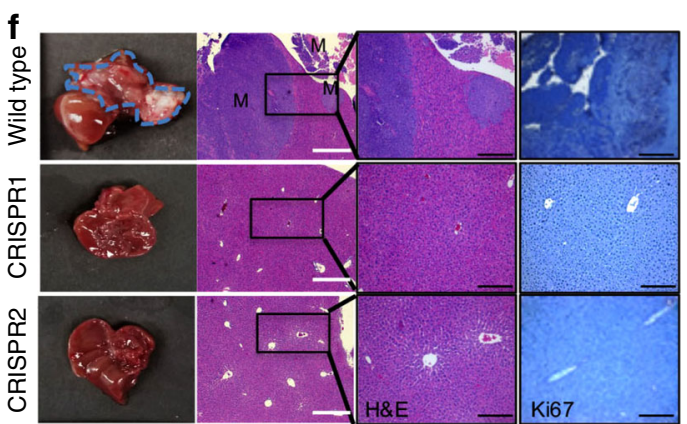

Fig. 5 RAMS11 induces liver metastasis via hemisplenectomy mouse model. a Representative mice showing no liver metastasis in RAMS11 CRISPR KO cell-injected mice by BLI. b RAMS11 CRISPR KO cell-injected mice show a significant decrease in liver metastasis by day 21 . c Day 21 ex vivo mouse livers show decreased metastasis in RAMS11 CRISPR KO cell-injected mice by BLI. Wild-type cell-injected mice had $\mathbf{d}$ increased liver weights and $\mathbf{e}$ liver metastasis compared with CRISPR KO cell-injected mice. $\mathbf{f}$ Hematoxylin and eosin stain of livers showing metastasis (M) and levels of Ki67 stain. Three independent tissues were stained per group. White bar $=10 \mu \mathrm{M}$, black bar $=100 \mu \mathrm{M}$. Data shown as mean \pm SEM and analyzed by two-tailed paired $t$-test, with WT $n=18$, CRISPR1 $n=11$, CRISPR2 $n=11$ per group, experiment was repeated three times. ${ }^{\star} p<0.05$, ${ }^{\star *} p<0.005$, ${ }^{\#} p<0.0005$. Source data are provided as a Source Data File.

overexpression promoted resistance to topoisomerase inhibitors, we narrowed our focus on clinically relevant drugs that selectively target the DNA topoisomerase TOP $2 \alpha$, doxorubicin and epirubicin. Measuring cellular viability in LoVo RAMS11 CRISPR KO cells we showed a 1.5-fold increase in drug sensitivity with $0.5 \mu \mathrm{M}$ doxorubicin or $0.7 \mu \mathrm{M}$ epirubicin treatment compared with wild-type treated cells $(p<0.05$, two-tailed paired $t$-test, Fig. 6c).

To further support our drug panel findings, we evaluated whether RAMS11 regulated TOP $2 \alpha$ protein expression. We observed that RAMS11 overexpressing cell lines had elevated TOP $2 \alpha$ protein expression, whereas our CRISPR KO cells displayed a decrease in TOP $2 \alpha$ protein levels (Fig. 6d). The decrease in TOP2 $\alpha$ expression in our CRISPR KO cells was rescued by re-introducing RAMS11 expression in these cells (Fig. 6d). Transient silencing of RAMS11 in SW620 cells also decreased TOP $2 \alpha$ protein and mRNA levels relative to our scrambled control (Supplementary Fig. 6a, b). To demonstrate the specificity of RAMS11 regulation of TOP $2 \alpha$, we confirmed there was only a decrease of TOP $2 \alpha$ mRNA expression by making primers specifically targeting TOP $2 \alpha$ and not TOP $2 \beta$ in the LoVo CRISPR KO and SW620 silenced cell lines
(Supplementary Fig. 6b, c). Lastly, we assessed downstream targets of TOP $2 \alpha$ in our CRISPR KO cell lines and observed a decrease in MLH1 and ERCC2 mRNA levels supporting RAMS11 regulation of TOP $2 \alpha$ (Supplementary Fig. 6d). Overall, our highthroughput drug panel established that RAMS11 expression impacted cellular sensitivity to topoisomerase inhibitors, specifically inhibitors targeting TOP $2 \alpha$, which led to the discovery that RAMS11 overexpression increased TOP $2 \alpha$ protein expression in colon cancer cell lines. These data highlight the potential for RAMS11 expression to serve as an important biomarker to select mCRC patients that may potentially benefit from topoisomerase inhibitor treatment.

RAMS11 binds to chromobox protein 4 (CBX4) to regulate TOP2 $\alpha$. Due to the nuclear localization of RAMS11 we hypothesized that it may transcriptionally regulate $T O P 2 \alpha$ expression to increase protein levels and promote topoisomerase resistance. Notably, a recent study found that CBX4 bound to the promoter of Top $2 \alpha^{45}$. Since CBX4 is known to possess both activation and repressive activities 46,47 , and has been found to interact with lncRNAs ${ }^{47,48}$, we hypothesized that it could interact with RAMS11 
a

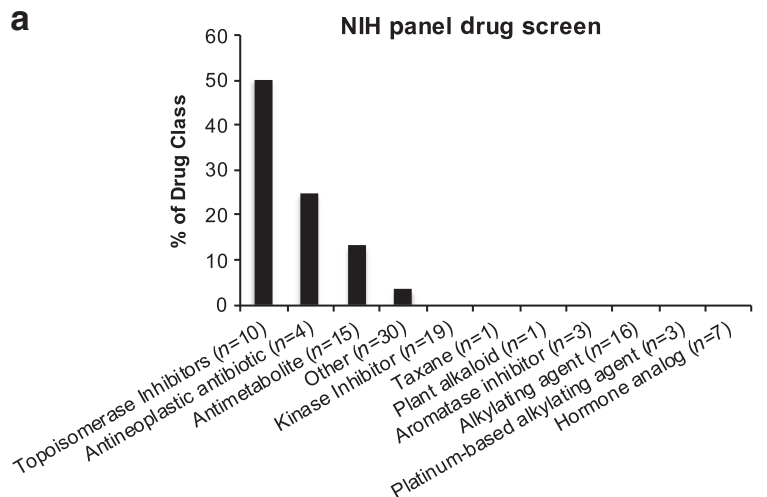

c

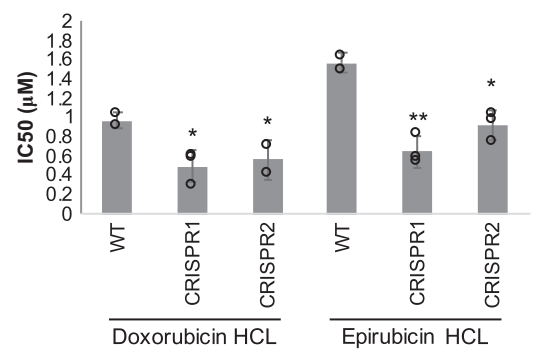

b

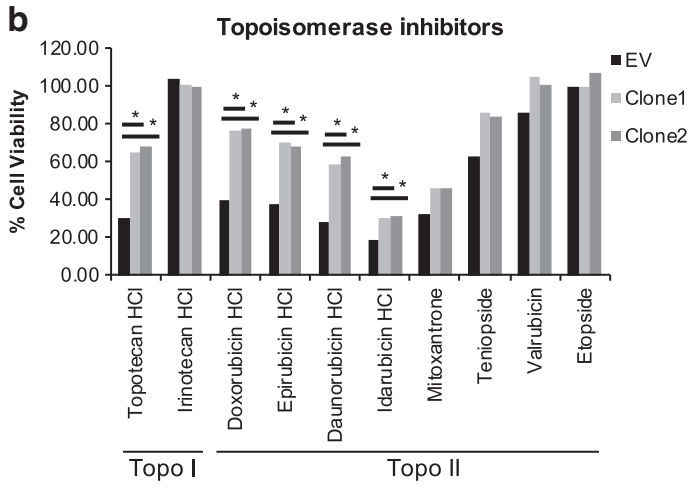

d

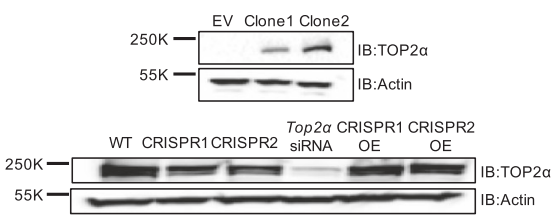

Fig. 6 RAMS11 expression alters sensitivity to topoisomerase inhibitors. a Cell viability assay comparing HT29 empty vector cells with RAMS11 overexpressing cell lines showing significant resistance to various drug classes. b RAMS11 overexpressing cells have increased cell viability compared with empty vector cells in five of ten topoisomerase (Topo) inhibitors. c IC50 values of RAMS11 CRISPR KO cell lines $(n=3)$ with decreased viability to doxorubicin hydrochloride $(\mathrm{HCl})$ and epirubicin $\mathrm{HCl}$ drug treatments. d Protein expression of Top2 $\alpha$ in (top) RAMS11 overexpressing cell lines and (bottom) CRISPR KO cell lines, TOP2 $\alpha$ siRNA control cells, and CRISPR cell lines overexpressing (OE) RAMS11. Band intensities were quantified from the digital image in Image J and are shown normalized to the empty vector or wild-type lane for each target. Samples derived from the same experiment and blots were processed in parallel. All data are presented as mean values \pm s.d. Experiments repeated three times. ${ }^{\star}$ Fold change $>1.5$. Source data are provided as a Source Data File.

and transcriptionally regulate $T O P 2 \alpha$ expression through interaction with CBX4. We found an 83-fold and 16-fold enrichment of RAMS11 bound to CBX4 in LoVo and SW620 cells, respectively, as determined by an RNA immunoprecipitation (RIP) coupled with qPCR (Fig. 7a, b). To orthogonally validate these findings, we conducted a RNA pull-down assay utilizing a $5^{\prime}$ Bromo-UTP fulllength RAMS11 sense labeled probe and a negative control antisense probe to pull-down proteins that may be bound to RAMS11. We found that the RAMS11 sense probe was bound to CBX4 protein compared with the antisense probe (Fig. 7c) by Western blot of nuclear lysates. In order to identify the regions of RAMS11 that bind to CBX4, we conducted in vitro RNA pull down in the LoVo cell line using four truncated RAMS11 fragments (Supplementary Fig. 7a). We re-validated our previous findings that fulllength RAMS11 binds to CBX4 and revealed that nucleotides 600-959 of RAMS11 interact with CBX4 protein (Supplementary Fig. 7b). These orthogonal methods support RAMS11 binding to CBX4 protein.

We next evaluated if CBX4 interacts with the TOP $2 \alpha$ promoter and whether this was dependent on RAMS11 expression. Binding of CBX4 to the promoter of TOP $2 \alpha$ was confirmed by chromatin immunoprecipitation (ChIP) coupled with qPCR in LoVo colon cancer cells. Silencing CBX4 led to a $78 \%$ decrease in CBX4 occupancy at the TOP $2 \alpha$ promoter in LoVo cells $(p=0.021$, twotailed paired $t$-test, Fig. $7 \mathrm{~d})$. Further, we demonstrated this binding is dependent on RAMS11 expression since there was a greater than $68 \%$ decrease in CBX4 occupancy in the TOP $2 \alpha$ promoter in our RAMS11 CRISPR KO models $(p<0.005$, two-tailed paired $t$-test, Fig. $7 \mathrm{~d}$ ). We also observed a decrease in tri-methylation of lysine 4 on the Histone $\mathrm{H} 3$ protein subunit (H3K4me3), a modification commonly associated with active transcription, in CBX4 siRNA treated cells and our RAMS11
CRISPR KO models (CRISPR1 $p=0.001$ and CRISPR2 $p=$ 0.0008 , two-tailed paired $t$-test, Fig. 7e). Decreased CBX4 occupancy and H3K4me3 at the TOP $2 \alpha$ promoter was further confirmed in the SW620 cell line with transiently silenced CBX4 or RAMS11 (CBX4 $p=0.004$, RAMS11 siRNA $p<0.05$, twotailed paired $t$-test, Fig. $7 \mathrm{f}, \mathrm{g})$. In addition to demonstrating endogenous binding of RAMS11 to CBX4 in LoVo and SW620 cells, we found more than 15000-fold enrichment of CBX4 binding to RAMS11 in our HT29 RAMS11 overexpressing cells compared with empty vector (Fig. 7h). In addition, the HT29 RAMS11 overexpressing cells had increased occupancy of CBX4 ( $p=0.0005$ and $p=0.004$, two-tailed paired $t$-test) and increased occupancy of $\mathrm{H} 3 \mathrm{~K} 4 \mathrm{me} 3(p=0.01$ and $p=0.001$, two-tailed paired $t$-test) at the TOP $2 \alpha$ promoter (Fig. $7 \mathrm{i}, \mathrm{j}$ ). Further, we rescued $\mathrm{CBX} 4$ (Fig. 7k) and $\mathrm{H} 3 \mathrm{~K} 4 \mathrm{me} 3(p=0.005$, two-tailed paired $t$-test, Fig. 7l) occupancy by re-introducing RAMS11 expression into the LoVo CRISPR KO cells. The decrease in TOP2 $\alpha$ protein expression was confirmed by Western blot in RAMS11 CRISPR KO and CBX4 silenced cells (Fig. 7m). We also observed a decrease in cellular invasion when $C B X 4$ or TOP $2 \alpha$ were transiently silenced in LoVo cell lines (Supplementary Fig. 8a, b). Collectively, these data demonstrate RAMS11dependent CBX4 binding to the TOP $2 \alpha$ promoter. Taken together, we provide evidence of RAMS11-dependent CBX4 regulation of TOP $2 \alpha$ to induce the metastatic phenotype in CRC (Fig. 8).

\section{Discussion}

In the current study we performed transcriptome sequencing of matched normal, primary, and distant metastatic patient samples to identify lncRNAs associated with metastatic progression that 

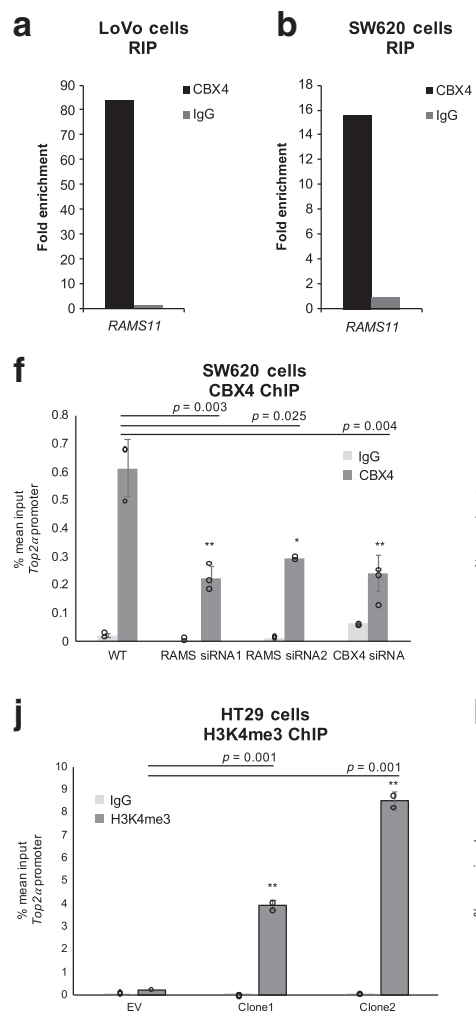
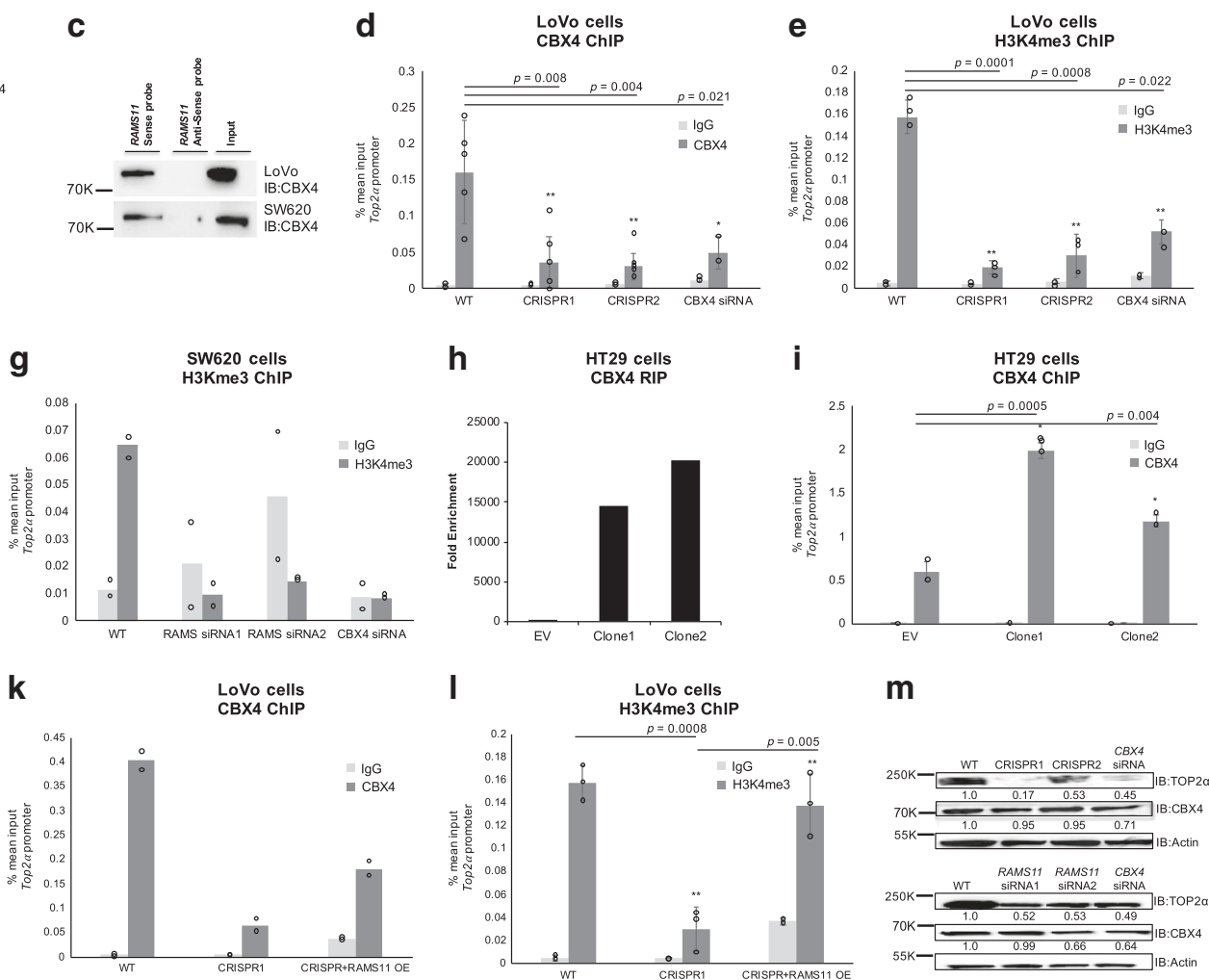

m

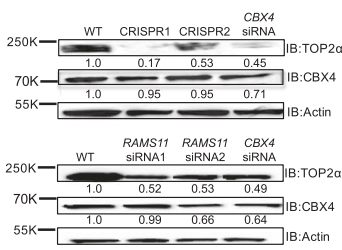

Fig. 7 RAMS11 binds to chromobox 4 (CBX4) to regulate expression of Top2 $\alpha$ mRNA and protein. RNA immunoprecipitation (RIP) shows binding of RAMS11 to CBX4 and not negative control IgG in a LoVo and $\mathbf{b}$ SW620 cells. $\mathbf{c}$ RNA pull down of 5-Bromo-UTP full-length RAMS11 probe showing binding of CBX4 by Western blot in LoVo and SW62O cells. $\mathbf{d}-\mathbf{g}$ Decreased binding of CBX4 and active histone mark H3K4me3 at TOP2 $\alpha$ promoter with silenced RAMS11 expression in chromatin immunoprecipitation (ChIP) assay. IgG $n=2, \mathrm{CBX} 4 n>3, \mathrm{H} 3 \mathrm{~K} 4 \mathrm{me} 3 n>2$. $\mathbf{h}$ RIP showing increased binding of $R A M S 11$ to CBX4 in HT29 RAMS11 overexpressing cells. i, j ChIP of CBX4 and H3K4me3 shows increased binding to TOP2 $\alpha$ promoter in HT29 RAMS11 overexpressing cells. IgG $n=3$, CBX4 $n=3, \mathrm{H} 3 \mathrm{~K} 4 \mathrm{me} 3 n=2$. $\mathbf{k}$, I ChIP of CBX4 and H3K4me3 in CRISPR KO cells with RAMS11 overexpression (OE) rescue at TOP2 $\alpha$ promoter. $\operatorname{lgG} n=2, \mathrm{CBX} 4 n=2, \mathrm{H} 3 \mathrm{~K} 4 \mathrm{me} 3 n=3$. $\mathbf{m}$ Protein expression of TOP2 $\alpha$ and CBX4 in LoVo (top) and SW620 cell lines (bottom). Band intensities were quantified from the digital image in ImageJ and are shown normalized to the wild-type lane for each target. Samples derived from the same experiment and blots were processed in parallel. Fold change normalized expression to actin is shown below gel. All data are presented as mean values \pm s.d, analyzed by two-tailed paired $t$-test. Experiments repeated more than two times. ${ }^{\star} p<0.05{ }^{\star *} p>0.005,{ }^{*} p<0.0005$. Source data are provided as a Source Data File.

could serve as a resource for further functional characterization and biomarker studies. To exemplify this, we prioritized RAMS11 since it was overexpressed in primary and mCRC tumors and its expression correlated with poor disease-free survival. This demonstrates the potential utility of RAMS11 expression as a marker to stratify high-risk patients. Supporting our clinical findings, we were able to confirm that RAMS11 promoted oncogenic phenotypes in vitro and in vivo in several cancer types.

To assess the clinical potential of RAMS11 and elucidate its regulatory mechanism for promoting aggressive phenotypes we used a high-throughput drug assay. We found that antimetabolites gemcitabine and floxuridine had the most significant increase in cellular viability when RAMS11 was overexpressed. We also found that RAMS11 promoted resistance to more than half of the topoisomerase inhibitors screened. Currently, the elevated expression of TOP2 $\alpha$ in primary and mCRC patients ${ }^{49-51}$ has served as the rationale for using anthracylines to treat select patients with mCRC. This can be exemplified by an ongoing phase II study to investigate the efficacy of epirubicin as a second-line treatment for patients with TOP2 $\alpha$ gene amplification and oxaliplatin-refractory mCRC ${ }^{52}$ (EudraCT 2013-001648-79). Our study provides mechanistic insight into RAMS11-dependent TOP2 $\alpha$ regulation in $\mathrm{mCRC}$ to promote resistance to these inhibitors. In addition, despite the promise of using anthracylines as a mCRC treatment, there are still many limitations including dose-limiting toxicities, intestinal toxicities, cumulative cardiotoxicity, and off-target effects on TOP $2 \beta$ leading to TOP $2 \beta$ poisoning 53 . Fortuitously, RAMS11 specifically targets TOP $2 \alpha$, and could be investigated for its therapeutic potential given the increased use of RNA therapeutics, such as locked nucleic acids, in clinical trials.

Currently, several topoisomerase inhibitors are currently FDA approved for treating multiple cancer types and are first-line therapies for breast cancer, bone and soft tissue sarcoma, bladder cancer, anaplastic thyroid cancer, Hodgkin's and non-Hodgkin's lymphoma, and multiple myeloma ${ }^{53-55}$. In addition, TOP2 $\alpha$ is used as a proliferation marker in multiple cancer types, including $\mathrm{CRC}^{56,57}$, and elevated levels of TOP2 $\alpha$ expression are associated with metastasis in prostate cancer, pancreatic cancer, and breast cancer ${ }^{58-61}$. Therefore, the clinical impact of our study extends beyond $\mathrm{MCRC}$ and affects a broader patient population given the widespread use of FDA-approved topoisomerase inhibitors coupled with the altered expression of RAMS11 across multiple solid tumors.

Overall, our understanding of how lncRNAs promote metastasis in CRC patients may have tremendous biological and clinical significance. To address this, our study used patient samples to characterize the landscape of lncRNA expression throughout the progression of primary to mCRC. We also show that lncRNA 


\section{Metastatic colorectal cancer patient samples}

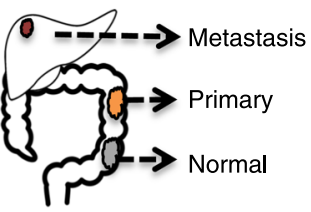

Whole transcriptome sequencing to identify all long non-coding RNAs
RAMS11

induces an invasive phenotype
RAMS11 binds to CBX4 to promote invasive phenotype by upregulating expression of Top2a

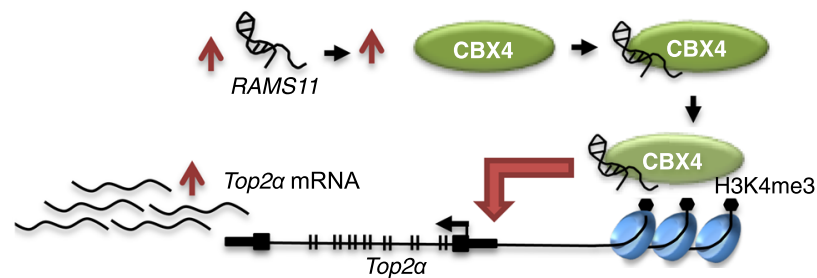

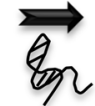

RAMS11 most up-regulated mCRC IncRNA

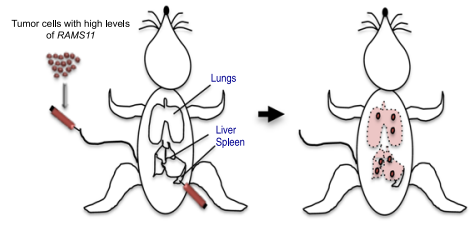

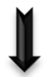

High throughput drug viability assay used to determine mechanism of RAMS11

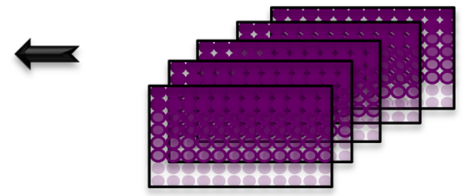

Fig. 8 RAMS11 identification and model in metastatic colorectal cancer. Process of identifying RAMS11 and model showing RAMS11 CBX4 complex binding to Top2 $\alpha$ promoter to increase metastatic phenotype.

RAMS11 directly affects mCRC biology, including promoting an aggressive phenotype and correlating with treatment response and resistance.

\section{Methods}

Patient samples and RNA sequencing. Patients were enrolled at Washington University School of Medicine in St. Louis and informed consent was obtained under an IRB-approved protocol. Adjacent normal, primary, and liver metastasis tissues were resected from mCRC patients and fresh frozen prior to RNA extraction (Supplementary Data 4). PolyA cDNA libraries were constructed using NuGen Ovation Kit V2, and paired-end sequencing was performed on Illumina HiSeq 2000. RNA-Seq data from Kim cohort was downloaded from NCBI GEO (GSE50760). TCGA RNA-Seq pre-aligned bam files were downloaded from the Cancer Genomics Hub (http://cghub.ucsc.edu/).

RNA-Seq data analysis. The human reference genome assembly version GRCh38/ hg38 and the corresponding gene annotations were used in RNA-Seq analysis. Gene annotations were combined from Gencode v23 ${ }^{62}$, RefSeq downloaded from the UCSC Genome Browser ${ }^{63}$, and the Broad lncRNA catalog ${ }^{64}$. Redundant transcripts were removed and overlapping transcripts were assigned to the same gene. RNA-Seq reads were aligned to the human genome using Tophat $2.0 .8^{65}$. Transcript assemblies were generated using Cufflinks 2.1.166. FeatureCounts v1.5.067 was used to generate fragment counts for individual transcripts requiring a mapping quality score $\geq 1$. FPKM was calculated using transcript fragment counts. For DE analysis, the transcript with highest FPKM among isoforms were selected to represent a gene locus, similar to our previous approach ${ }^{68}$. EdgeR 3.8.6 $6^{69}$ was used to perform a TMM normalization and DE analysis using the raw fragment counts. In the meta-analysis, DE $p$ values were combined using the Stouffer method $^{70}$ and fold change was averaged between WUSTL and Kim cohorts. RAMS were defined as IncRNAs that were not tissue specific and DE between metastasis versus primary tumor and between metastasis versus normal tissue $(\mathrm{FC} \geq 2, \mathrm{FDR} \leq 0.05)$.

Exon array data analysis. We repurposed the Affymetrix exon array for lncRNA analysis by realigning the probe set sequences against the human transcript sequences using SeqMap 1.0.12 $2^{71}$ allowing one mismatch. Only probe sets consisting of probes that were uniquely aligned to transcripts from the same gene were retained. Exon array expression was processed and normalized using Affymetrix Power Tool 1.18 (https://www.thermofisher.com).
Survival analysis. Survival analysis was performed using the Cox proportional hazard model with R survival package 2.37-7 2014 (https://CRAN.R-project.org/ package $=$ survival). The median expression of RAMS11 within a cohort was used to stratifying patients into low and high RAMS11 expression groups. Kaplan-Meier curves were plotted using the R survplot package 0.0.7 2014 (http://www.cbs.dtu. $\mathrm{dk} / \sim$ eklund/survplot/).

Cell culture. Colon cancer cell lines CCD18-Co and SW480 were a kind gift from Dr David Shalloway at Cornell University. All other colon cell lines (HT29, HT-15, DLD1, SW620, Caco-2, HCT-116, and RKO) were a kind gift from Dr A. Craig Lockhart at Washington University. LoVo cell lines were purchased from ATCC (ATCC CCL-229). HCC95 and A549 cell lines were a kind gift from Dr Lauren Michel and Dr Brian Van Tine, respectively, from Washington University. SW620 cells were grown in DMEM (Invitrogen, Carlsbad, CA) with $10 \%$ fetal bovine serum (Sigma, St. Louis, MO), and 1\% penicillin/streptomycin (Invitrogen, Carlsbad, CA) complete media. LoVo cells were grown in DMEM/F12 (Invitrogen) with $10 \%$ fetal bovine serum, and $1 \%$ penicillin/streptomycin complete media. HT29, HT-15, DLD1, and Caco-2 cells were grown in McCoys (Invitrogen) with $10 \%$ fetal bovine serum and $1 \%$ penicillin/streptomycin, and all other cells were grown in RPMI (Invitrogen) with 10\% fetal bovine serum, and 1\% penicillin/ streptomycin complete media.

Rapid amplification of CDNA ends (RACE). $5^{\prime}$ and $3^{\prime}$ RACE was done using the GeneRacer Kit (Invitrogen) according to the manufacturer's instructions. RACE PCR products were obtained with Platinum Taq High Fidelity (Invitrogen) using the GeneRacer primer (supplied) and a gene-specific primer found in Supplementary Data 5 . Products were visualized on a $2 \%$ agarose gel and purified by gel extraction (Qiagen, Germantown, MD). This product was then cloned into pcr4TOPO vector (Invitrogen) and grown in TOP10 E. coli. Clones were sequenced with the M13 forward primer at the Protein and Nuclei Acid Chemistry Laboratory at Washington University.

Generation of RAMS11 silenced and overexpression cells. LoVo RAMS11 CRISPR KO cell lines were generated through the Genome Engineering and iPSC center at the Washington University. CRISPR/Cas9 was used to create a genomic deletion of the last four exons of RAMS11 in LoVo metastatic colon cancer cells (Supplementary Fig. 2a). We used two different cell clones for the described experiments. In addition, we silenced expression of RAMS11 using custom silencer select RNAs (siRNAs) targeting RAMS11, CBX4, TOP2 $\alpha$, or a negative scrambled control (Invitrogen). siRNA sequences are listed in Supplementary Data 5.

Full-length RAMS11 transcript was PCR amplified from LoVo cells and cloned into the pCFG5-IEGZ vector (a kind gift from Dr Ron Bose, Washington University). Full-length RAMS11 inserts were confirmed with Sanger sequencing at 
the Protein and Nuclei Acid Chemistry Laboratory at Washington University. Retroviral infection of cancer cells was performed according to Kauri et al. ${ }^{72}$. Briefly, the amyotrophic phoenix cell line was transfected with $10 \mu \mathrm{g}$ of pCFG5RAMS11 or empty vector control by calcium phosphate precipitation and incubated for $24 \mathrm{~h}$. Viral supernatants were harvested after an additional 24-h incubation. Virus was added to cells seeded in six-well dishes in the presence of 8 $\mu \mathrm{g} / \mathrm{mL}$ polybrene (Sigma). Cells were centrifuged at $300 \times g$ for $90 \mathrm{~min}$ and fresh media was added to the plate. After 14 days of Zeocin (Invitrogen) selection cells were used for assays. HT29 colon cells that had low endogenous expression of RAMS11 were infected with virus expressing RAMS11 or empty vector for $48 \mathrm{~h}$ and selected with $100 \mu \mathrm{g} / \mathrm{mL}$ Zeocin.

Quantitative real-time PCR. Total RNA was isolated for each CRC cell line using Takara Bio NucleoSpin RNA (Takara, Mountain View, CA). Total RNA was then transcribed to cDNA with SuperScript III First strand cDNA system (Invitrogen) and quantified using Fast Sybr Green Master Mix (Invitrogen) as per the manufacturer's protocol. Primer sequences are available in Supplementary Data 5.

Protein detection by Western blot. Western blots were conducted by plating 250,000 representative cancer cells in a six-well dish. For transient knockdown experiments, the next day cells were transfected at $6.25-25 \mathrm{nM}$ with two independent custom designed siRNAs or a negative scramble control with Lipofectamine RNAiMax (Invitrogen) for $72 \mathrm{~h}$. Cells were then lysed with Tris lysis buffer $(50 \mathrm{mM}$ Tris- $\mathrm{HCl}, 1 \%$ Triton X-100, $131 \mathrm{mM} \mathrm{NaCl}, 1 \mathrm{mM}$ sodium orthovanadate, $10 \mathrm{mM} \mathrm{Na}_{4} \mathrm{P}_{2} 0_{7}, 10 \mathrm{mM} \mathrm{NaF}, 1 \mathrm{mM}$ EDTA), run on NuPAGE $4-12 \%$ Bis-Tris gel (Invitrogen) and transferred to nitrocellulose membrane (BioRad, Hercules, CA). Blots were then probed overnight at $4^{\circ}$ with respective antibodies including TOP2A, CBX4, and ACTIN, then washed with TBST buffer, and then applied with secondary goat anti-rabbit HRP-linked or goat anti-mouse HRP-linked antibodies (Thermo Fisher, Waltham, MA). Blots were then washed, visualized with Clarity Western ECL Substrate (Bio-Rad), and imaged using the ChemiDoc XRS+ System (Bio-Rad). Band intensities were quantified from the digital image in ImageJ and are shown normalized to the control lane for each target. Raw Western blots are shown in Supplementary Fig. 9. All antibodies and concentrations are listed in Supplementary Data 6.

RNA immunoprecipitation. RIP coupled to $\mathrm{qPCR}$ assays were conducted by isolating nuclear lysates from ten million LoVo or SW620 cells following the NERPER Nuclear and Cytoplasmic Extraction Reagent Kit (Thermo Fisher). Nuclear lysates were then incubated overnight with $5 \mu \mathrm{g}$ CBX4 antibody or IgG antibody

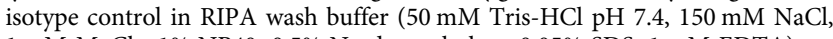
$1 \mathrm{mM} \mathrm{MgCl} 2,1 \%$ NP40, $0.5 \%$ Na-deoxycholate, $0.05 \%$ SDS, $1 \mathrm{mM} \mathrm{EDTA}$ ) and SUPERase-in RNAse inhibitor (Invitrogen). The next day $50 \mu \mathrm{L}$ of Invitrogen Dynabeads Protein $G$ were added to the antibody lysate/mixture and were rotated for $1 \mathrm{~h}$ at $4^{\circ}$. Next, beads were subsequently washed six times with RIPA wash buffer using a magnetic bead separator. Protein was then digested with proteinase $\mathrm{K}$ buffer (RIPA buffer, $10 \% \mathrm{SDS}, 10 \mathrm{mg} / \mathrm{mL}$ proteinase $\mathrm{K}$ ), at $55^{\circ}$ for $30 \mathrm{~min}$ shaking. RNA was phenol:chloroform:isoamyl alcohol extracted following the general protocol (Thermo Fisher). Last, gDNA was removed from RNA using ArcticZymes Heat and Run gDNA removal kit following the manufacturer's protocol (Tromso, Norway). cDNA was made using SuperScript III First strand cDNA system as indicated above and qPCR was run with Fast Sybr Green Master Mix and indicated primers (Supplementary Data 5). Fold enrichment of qPCR results were calculated following Sigma-Aldrich Data Analysis Calculation Shell by comparing nonspecific control IgG antibody raw CTs to CBX4 RNA binding protein CT normalized against $1 \%$ input.

Chromatin immunoprecipitation. ChIP qPCR assays were conducted by first sonicating five million cells in SDS lysis buffer (1\% SDS, $500 \mathrm{mM}$ EDTA, $50 \mathrm{mM}$ Tris-HCl pH 8). Next, sonicated cells were immunoprecipitated with $5 \mu \mathrm{g}$ IgG, CBX4, or H3K4me3 antibodies in ChIP dilution buffer $(0.01 \%$ SDS, $1.10 \%$ Triton X-100, $1.2 \mathrm{nM}$ EDTA, $16.7 \mathrm{mM}$ Tris- $\mathrm{HCl} \mathrm{pH} 8,167 \mathrm{mM} \mathrm{NaCl}$ ), and $1 \mathrm{X}$ Halt Protease and Phosphatase inhibitors overnight with rotation at $4^{\circ}$. The next day Dynabeads Protein G (Invitrogen) were added to the antibody lysate mixture and rotated for $1 \mathrm{~h}$. Bead/lysate mixture was then washed once with low salt wash buffer (0.1\% SDS, $1 \%$ Triton X-100, 2 mM EDTA, $20 \mathrm{mM}$ Tris-HCl pH $8,150 \mathrm{mM} \mathrm{NaCl})$, then high salt buffer $(0.1 \%$ SDS, $1 \%$ Triton X-100, 2 mM EDTA, $20 \mathrm{mM}$ Tris- $\mathrm{HCl}$ $\mathrm{pH} 8,500 \mathrm{mM} \mathrm{NaCl})$, lithium chloride wash buffer $(0.25 \mathrm{M}$ lithium chloride, $1 \%$ NP40, $1 \%$ sodium deoxycholate, $1 \mathrm{mM}$ EDTA, $10 \mathrm{mM}$ Tris- $\mathrm{HCl} \mathrm{pH} \mathrm{8),} \mathrm{and} \mathrm{finally}$ two washes with Tris-HCl EDTA buffer (10 mM Tris-HCl pH 8, 1 mM EDTA). DNA was eluted by incubating beads for $30 \mathrm{~min}$ at room temperature with SDS elution buffer (1\% SDS, $0.1 \mathrm{M}$ sodium bicarbonate), followed by $1.25 \mathrm{M} \mathrm{NaCl}$ and $2.5 \mathrm{mg} / \mathrm{mL}$ RNAse A at $95^{\circ}$ for $15 \mathrm{~min}$ shaking followed by addition of proteinase K buffer $(1 \mu \mathrm{L} 10 \mathrm{mg} / \mathrm{mL}$ proteinase $\mathrm{K}, 5 \mu \mathrm{L} 0.5 \mu \mathrm{M}$ EDTA, $10 \mu \mathrm{L} 1 \mathrm{M}$ Tris $\mathrm{pH} 7.5)$ shaking at $60^{\circ}$ for $15 \mathrm{~min}$. DNA was then isolated using phenol:chloroform:isoamyl alcohol extraction following the general protocol as mentioned above. DNA was diluted by five and used for qPCR. The \% input calculations were determined by comparing CT values from input DNA and ChIP DNA for the TOP2A target promoter region using the following equation: \%Input $=\%$ of starting input fraction $\times 2^{[C T(\text { input) }-\mathrm{CT}(\mathrm{ChIP})]}$. Primer sequences are available in Supplementary Data 5 .

BrU-labeled RNA pull down. Full-length RNA probes and fragmented RAMS11 probes were made using the Promega Riboprobe in vitro transcription kit from $2.5 \mu \mathrm{g}$ of linearized DNA in the pGEM-3Z vector (Madison, WI). Antisense probes were made by in vitro transcription from the SP6 promoter. RAMS11 RNA pulldown experiments were performed in LoVo and SW620 nuclear lysates following the RiboTrap Kit manufacturer's protocol (MBL, Woburn, MA). Truncated RAMS11 probes consisted of fragments $1-250,200-450,400-650$, and 600-959 basepairs of RAMS11. RAMS11 probes were synthesized and subcloned at Gene Universal (Newark, DE).

Nuclear cytoplasmic isolations. Nuclear and cytoplasmic isolations were conducted using the PARIS Kit (Thermo Fisher) following the manufacturer's protocol. Total RNA was also collected as described above. Nuclear and cytoplasmic isolations were calculated by normalizing respective gene to total RNA expression.

Transwell assays. Cell lines were seeded at 300,000 cells in a six-well dish. The next day cells were transfected with siRNAs targeting RAMS11, TOP2 $\alpha$, CBX4, or overexpression plasmids. Seventy-two hours later cells were harvested and reseeded at 200,000 cells on a transwell $8.0 \mu \mathrm{M}$ permeable membrane support (Corning, Corning, NY) in 24-well plates for a modified Boyden chamber assay. A serum gradient was established with cells plated in serum-free media and complete media (10\% FBS) added to the bottom of the well. For invasion assays, transwells were precoated with $200 \mu \mathrm{g} / \mathrm{mL}$ Matrigel (Corning) before addition of cells. Cells were allowed to migrate or invade overnight and then fixed with $4 \%$ paraformaldehyde (Electron Microscopy Sciences, Hatfield, PA), and nuclei were stained with DAPI (Sigma, $1 \mu \mathrm{g} / \mu \mathrm{L}$ ). A cotton swab was used to remove cells from the top of the membrane. Migrated DAPI-stained cells were imaged with Q-Capture Pro software on an Olympus IX70 microscope, quantified using ImageJ software (http://imagej. nih.gov/ij/), and statistical significance was determined by a Student's two-tailed $t$-test. Four to seven images were taken per transwell membrane at $\times 20$ magnification. Assays were repeated two to three times.

Soft agar assays. HT29 cells overexpressing RAMS11 and LoVo RAMS11 CRISPR KO cell lines were resuspended at 75,000 cells in $0.4 \%$ Difco soft agar (BD Bioscience, Franklin Lakes, NJ) and seeded onto a 5\% Difco base agar. Cells were given fresh media every 3 days for around 2 weeks, and once colonies were visible by eye, cells were stained with $0.5 \%$ Crystal Violet (Sigma) for $3 \mathrm{~h}$. Plates were then imaged using the ChemiDoc XRS+ (Bio-Rad) and counted with ImageJ software. Average cell counts were used for comparison and statistical significance was determined by a Student's two-tailed $t$-test. Assays were repeated three times.

Drug treatments. The NIH approved oncology library of 119 drugs (AOD6 plate 4825-1 and AOD6 plate 4826) was received from the NIH National Cancer Institute DTP Developmental Therapeutics Program. Drugs were diluted in DMSO to $1 \mathrm{mM}$ and the well assignments were rearranged so drugs were confined to the inner 60 wells of 96-well plates. HT29 RAMS11 overexpressing Clone1 and Clone2 cell lines were seeded at 5000 cells per well in a 96 -well plate. The next day serial diluted drug was added to pre-seeded plates in media containing $1 \%$ DMSO vehicle, using a Robbins Hydra 96 microdispenser. Two 96-well plates of cells were used as vehicle controls. The plates were incubated for 3 days. Percent viability was scored by incubating cells for $3 \mathrm{~h}$ with resazurin sodium $(0.023 \mathrm{mg} / \mathrm{mL}$, Sigma R7017). The reaction was stopped by the addition of SDS ( $1 \%$ final concentration) Fluorescence Ex/Em 540/590 was read in a Biotek Synergy H1 plate reader (Winooski, VT). The fluorescence values for the vehicle plates were averaged and percent viability was determined by the formula: Percent viability $=($ average vehicle - value)/(average vehicle - average resazurin in media blank) $\times 100$. We removed drugs that were undetectable, or out of range, by resazurin assay, leaving 118 drugs to assess in the study (Supplementary Data 3). Values with more than a 1.5-fold change in both RAMS11 Clone1 and Clone2 overexpressing cell lines were used to determine significance. Individual drug IC50 assays were done in a similar manner as described above with CRISPR KO cells. Assays were repeated more than three times.

In vivo models. The animal studies were reviewed and approved by the Washington University's Institutional Animal Care and Use Committee protocol For subcutaneous injections, 2e6 LoVo wild-type, RAMS11 CRISPR1, or RAMS11 CRISPR2 luciferase-tagged cells were injected subcutaneously in ten NOD/SCID mice per group. Weekly tumor size was determined by caliper measurements comparing length $\times$ width $\times$ height $\times 0.5$. For post analysis lung tissues and subcutaneous tumors tissues were removed and formalin fixed and paraffin embedded. This experiment was repeated two times.

In the lung metastasis mouse model, 2e6 LoVo wild-type and CRISPR luciferase-tagged cell models were injected into the lateral tail vein of twelve 5-week-old NOD/SCID mice (Jackson Laboratories, Bar Harbor, MA) per group using 30-gauge needles. In weekly intervals mice were imaged with the Olympus 
OV100 Small Animal Imaging System (IVIS Spectrum, Caliper, Hopkinton, MA) in conjunction with the Small Animal Imaging Core (SAIC) at Washington University. Mice were imaged for $1 \mathrm{~min}$ with sequential 5-s exposures. Luminescence was quantified using the Living Image Software 3.2 (Caliper). All micrometastasis were imaged at day $0(30 \mathrm{~min}$ to $1 \mathrm{~h})$ post operation and weekly for 12 weeks. At the conclusion of the study, mice were sacrificed and examined visually and with bioluminescence for lung metastases in vivo and ex vivo. Lungs were dissected and formalin fixed and paraffin embedded for histological analysis with H\&E and Ki67 staining. This experiment was repeated twice.

For the hemisplenectomy mouse model ${ }^{38}, 2 \mathrm{e} 6$ LoVo wild-type and CRISPR luciferase-tagged cells in $50 \mu \mathrm{L}$ of PBS were injected into the spleen of 6-8-weekold NGS mice (Jackson Laboratories) (WT $n=18$, CRISPR1 $n=11$, CRISPR $2 n=$ 11) using 30-gauge needles during open laparotomy. Cell injections were followed with a $50 \mu \mathrm{L}$ PBS flush. Incisions were closed with sutures and surgical clips. In weekly intervals mice were imaged with the Olympus OV100 Small Animal Imaging System (IVIS Spectrum) in conjunction with SAIC. Mice were imaged for $10 \mathrm{~s}$ to $1 \mathrm{~min}$ exposures. Luminescence was quantified using the Living Image Software 3.2 (Caliper). All micrometastasis were imaged at day 7, day 14, and day 21 post operation. At the conclusion of the study, mice were sacrificed and examined visually and by bioluminescence for liver metastases in vivo and ex vivo. Livers were dissected, weighed, and formalin fixed and paraffin embedded for histological analysis with H\&E and Ki67 staining. This experiment was repeated three times.

Reporting summary. Further information on research design is available in the Nature Research Reporting Summary linked to this article.

\section{Data availability}

The RNA-Seq data generated in this study (WUSTL cohort) have been deposited in the $\mathrm{dbGaP}$ database under the accession code phs001722. The Kim et al.'s data ${ }^{29}$ referenced during the study are available in a public repository from the NCBI Gene Expression Omnibus under the accession code GSE50760. The source data underlying all figures are provided as a Source Data File. All the other data supporting the findings of this study are available within the article and its Supplementary Information files and from the corresponding author upon reasonable request. A reporting summary for this article is available as a Supplementary Information file.

Received: 31 October 2018; Accepted: 16 March 2020;

Published online: 01 May 2020

\section{References}

1. Siegel, R., Naishadham, D. \& Jemal, A. Cancer statistics, 2012. CA 62, 10-29 (2012).

2. Lee, J. J. \& Sun, W. Options for second-line treatment in metastatic colorectal cancer. Clin. Adv. Hematol. Oncol. 14, 46-54 (2016).

3. Siegel, R. L. et al. Colorectal cancer statistics, 2017. CA 67, 177-193 (2017).

4. Temraz, S., Mukherii, D., Alameddine, R. \& Shamseddine, A. Methods of overcoming treatment resistance in colorectal cancer. Crit. Rev. Oncol. Hemat. 89, 217-230 (2014).

5. Grothey, A., Marshall, J. L. \& Bekaii-Saab, T. Sequencing beyond the secondline setting in metastatic colorectal cancer. Clin. Adv. Hematol. Oncol. 17, 1-19 (2019).

6. Garajová, I. et al. The best overall response to the first-line but not to the second-line treatment correlates with outcome of metastatic right-sided and left-sided colon cancer. Annals of Oncology 28, 105-106 (2017).

7. Das, S., Ciombor, K. K., Haraldsdottir, S. \& Goldberg, R. M. Promising new agents for colorectal cancer. Curr. Treat. Options Oncol. 19, https://doi.org/ 10.1007/s11864-018-0543-z (2018).

8. Coppedè, F. Genetic and epigenetic biomarkers for diagnosis, prognosis and treatment of colorectal cancer. World J. Gastroenterol. 20, 943 (2014).

9. Yiu, A. J. \& Yiu, C. Y. Biomarkers in colorectal cancer. Anticancer Res. 36, 1093-1102 (2016).

10. Hibner, G., Kimsa-Furdzik, M. \& Francuz, T. Relevance of MicroRNAs as potential diagnostic and prognostic markers in colorectal cancer. Int. J. Mol. Sci. 19, 2944 (2018).

11. Rinn, J. L. \& Chang, H. Y. Genome regulation by long noncoding RNAs. Annu. Rev. Biochem. 81, 145-166 (2012).

12. Ling, H. et al. CCAT2, a novel noncoding RNA mapping to 8q24, underlies metastatic progression and chromosomal instability in colon cancer. Genome Res. 23, 1446-1461 (2013).

13. Weidle, U. H., Birzele, F., Kollmorgen, G. \& Ruger, R. Long non-coding RNAs and their role in metastasis. Cancer Genom. Proteom. 14, 143-160 (2017).

14. Gooding, A. J. et al. The IncRNA BORG drives breast cancer metastasis and disease recurrence. Sci. Rep. 7, 12698 (2017).
15. Li, H., Ma, S. Q., Huang, J., Chen, X. P. \& Zhou, H. H. Roles of long noncoding RNAs in colorectal cancer metastasis. Oncotarget 8, 39859-39876 (2017).

16. Silva, J. \& Smith, D. Long Non-Coding RNAs and Cancer. (Caister Academic Press, La Jolla, CA, 2012).

17. Silva, J. M., Boczek, N. J., Berres, M. W., Ma, X. \& Smith, D. I. LSINCT5 is over expressed in breast and ovarian cancer and affects cellular proliferation. RNA Biol. 8, 496-505 (2011).

18. Silva, J. M. et al. Identification of long stress-induced non-coding transcripts that have altered expression in cancer. Genomics 95, 355-362 (2010).

19. Sanchez, Y. \& Huarte, M. Long non-coding RNAs: challenges for diagnosis and therapies. Nucleic Acid Ther. 23, 15-20 (2013).

20. Ling, H., Fabbri, M. \& Calin, G. A. MicroRNAs and other non-coding RNAs as targets for anticancer drug development. Nat. Rev. Drug Discov. 12, 847-865 (2013).

21. Shi, D., Wu, F., Gao, F., Qing, X. \& Shao, Z. Prognostic value of long noncoding RNA CCAT1 expression in patients with cancer: a meta-analysis. PLoS ONE 12, e0179346 (2017).

22. Garajova, I. et al. Non-coding RNAs as predictive biomarkers to current treatment in metastatic colorectal cancer. Int. J. Mol. Sci. 18, 7 (2017).

23. Arriaga-Canon, C. et al. The use of long non-coding RNAs as prognostic biomarkers and therapeutic targets in prostate cancer. Oncotarget $\mathbf{9}$, 20872-20890 (2018).

24. Hon, K. W., Abu, N., Ab Mutalib, N. S. \& Jamal, R. miRNAs and lncRNAs as predictive biomarkers of response to FOLFOX therapy in colorectal cancer. Front. Pharmacol. 9, 846 (2018).

25. Cancer Genome Atlas Research, N. et al. The Cancer Genome Atlas pancancer analysis project. Nat. Genet. 45, 1113-1120 (2013).

26. Ouyang, S. et al. LncRNA BCAR4, targeting to miR-665/STAT3 signaling, maintains cancer stem cells stemness and promotes tumorigenicity in colorectal cancer. Cancer Cell Int. 19, 72 (2019).

27. Liu, Y., Zhang, M., Liang, L., Li, J. \& Chen, Y. X. Over-expression of lncRNA DANCR is associated with advanced tumor progression and poor prognosis in patients with colorectal cancer. Int J. Clin. Exp. Pathol. 8, 11480-11484 (2015).

28. Deng, H. et al. Long non-coding RNAs: New biomarkers for prognosis and diagnosis of colon cancer. Tumor Biology 39, https://doi.org/10.1177/ 1010428317706332 (2017).

29. Kim, S. K. et al. A nineteen gene-based risk score classifier predicts prognosis of colorectal cancer patients. Mol. Oncol. 8, 1653-1666 (2014).

30. Chen, X. et al. Integrated analysis of long non-coding RNAs in human colorectal cancer. Oncotarget 7, 23897-23908 (2016).

31. Kim, T. et al. Role of MYC-regulated long noncoding RNAs in cell cycle regulation and tumorigenesis. J. Natl Cancer Inst. 107, 4 (2015).

32. Matouk, I. J. et al. Highly upregulated in liver cancer noncoding RNA is overexpressed in hepatic colorectal metastasis. Eur. J. Gastroenterol. Hepatol. 21, 688-692 (2009)

33. Chen, S. W. et al. Overexpression of long non-coding RNA H19 is associated with unfavorable prognosis in patients with colorectal cancer and increased proliferation and migration in colon cancer cells. Oncol. Lett. 14, 2446-2452 (2017).

34. Schwarzenbach, H. Biological and clinical relevance of $\mathrm{H} 19$ in colorectal cancer patients. EBioMedicine 13, 9-10 (2016).

35. Sveen, A. et al. Transcriptome instability in colorectal cancer identified by exon microarray analyses: associations with splicing factor expression levels and patient survival. Genome Med. 3, 32 (2011).

36. Lee, B. et al. Long noncoding RNAs as putative biomarkers for prostate cancer detection. J. Mol. Diagn. 16, 615-626 (2014).

37. Cabanski, C. R. et al. Pan-cancer transcriptome analysis reveals long noncoding RNAs with conserved function. RNA Biol. 12, 628-642 (2015).

38. Grossman, J. G. et al. Recruitment of CCR2(+) tumor associated macrophage to sites of liver metastasis confers a poor prognosis in human colorectal cancer. Oncoimmunology 7, e1470729 (2018).

39. Jimenez-Fonseca, P. et al. Gemcitabine plus capecitabine (Gem-Cape) biweekly in chemorefractory metastatic colorectal cancer. Clin. Transl. Oncol. 17, 384-392 (2015).

40. Litvak, D. A. et al. Systemic irinotecan and regional floxuridine after hepatic cytoreduction in 185 patients with unresectable colorectal cancer metastases. Ann. Surg. Oncol. 9, 148-155 (2002).

41. Power, D. G. \& Kemeny, N. E. The role of floxuridine in metastatic liver disease. Mol. Cancer Ther. 8, 1015-1025 (2009).

42. Groot Koerkamp, B. et al. Perioperative hepatic arterial infusion pump chemotherapy is associated with longer survival after resection of colorectal liver metastases: a propensity score analysis. J. Clin. Oncol. 35, 1938-1944 (2017).

43. Khayat, D., Gil-Delgado, M., Antoine, E. C., Nizri, D. \& Bastian, G. The role of irinotecan and oxaliplatin in the treatment of advanced colorectal cancer. Oncology 15, 415-429 (2001).

44. Fernandes, G. D. S. et al. Combination of irinotecan, oxaliplatin and 5fluorouracil as a rechallenge regimen for heavily pretreated metastatic colorectal cancer patients. J. Gastrointest. Cancer 49, 470-475 (2018). 
45. Alekseyenko, A. A., Gorchakov, A. A., Kharchenko, P. V. \& Kuroda, M. I. Reciprocal interactions of human C10orf12 and C17orf96 with PRC2 revealed by BioTAP-XL cross-linking and affinity purification. Proc. Natl Acad. Sci. USA 111, 2488-2493 (2014).

46. Gil, J. \& O'Loghlen, A. PRC1 complex diversity: where is it taking us? Trends Cell Biol. 24, 632-641 (2014).

47. Yang, L. et al. ncRNA- and Pc2 methylation-dependent gene relocation between nuclear structures mediates gene activation programs. Cell 147, 773-788 (2011).

48. Bernstein, E. et al. Mouse polycomb proteins bind differentially to methylated histone $\mathrm{H} 3$ and RNA and are enriched in facultative heterochromatin. Mol. Cell. Biol. 26, 2560-2569 (2006).

49. Nygard, S. B. et al. Underpinning the repurposing of anthracyclines towards colorectal cancer: assessment of topoisomerase II alpha gene copy number alterations in colorectal cancer. Scand. J. Gastroenterol. 48, 1436-1443 (2013).

50. Sonderstrup, I. M. et al. Topoisomerase-1 and -2A gene copy numbers are elevated in mismatch repair-proficient colorectal cancers. Mol. Oncol. 9, 1207-1217 (2015).

51. Tsavaris, N. et al. Topoisomerase I and IIalpha protein expression in primary colorectal cancer and recurrences following 5-fluorouracil-based adjuvant chemotherapy. Cancer Chemother. Pharmacol. 64, 391-398 (2009).

52. Tarpgaard, L. S. et al. A phase II study of epirubicin in oxaliplatin-resistant patients with metastatic colorectal cancer and TOP2A gene amplification. BMC Cancer 16, 91 (2016).

53. Pommier, Y. Drugging topoisomerases: lessons and challenges. ACS Chem. Biol. 8, 82-95 (2013).

54. Bailly, C. Contemporary challenges in the design of topoisomerase II inhibitors for cancer chemotherapy. Chem. Rev. 112, 3611-3640 (2012).

55. Nitiss, J. L. DNA topoisomerases in cancer chemotherapy: using enzymes to generate selective DNA damage. Curr. Opin. Investig. Drugs 3, 1512-1516 (2002).

56. El-Deiry, W. S. et al. Molecular profiling of 6,892 colorectal cancer samples suggests different possible treatment options specific to metastatic sites. Cancer Biol. Ther. 16, 1726-1737 (2015).

57. Miura, J. T. et al. Tumor profiling of gastric and esophageal carcinoma reveal different treatment options. Cancer Biol. Ther. 16, 764-769 (2015).

58. $\mathrm{Li}, \mathrm{X}$. et al. TOP2Ahigh is the phenotype of recurrence and metastasis whereas TOP2Aneg cells represent cancer stem cells in prostate cancer. Oncotarget $\mathbf{5}$, 9498-9513 (2014).

59. Fountzilas, G. et al. Topoisomerase II alpha gene amplification is a favorable prognostic factor in patients with HER2-positive metastatic breast cancer treated with trastuzumab. J. Transl. Med. 10, 212 (2012).

60. Pei, Y. F., Yin, X. M. \& Liu, X. Q. TOP2A induces malignant character of pancreatic cancer through activating beta-catenin signaling pathway. Biochimica et Biophysica Acta 1864, 197-207 (2018).

61. Norimura, S. et al. Candidate biomarkers predictive of anthracycline and taxane efficacy against breast cancer. Cancer Res. Ther. 14, 409-415 (2018).

62. Harrow, J. et al. GENCODE: the reference human genome annotation for The ENCODE Project. Genome Res. 22, 1760-1774 (2012).

63. Speir, M. L. et al. The UCSC Genome Browser database: 2016 update. Nucleic Acids Res. 44, D717-D725 (2016).

64. Cabili, M. N. et al. Integrative annotation of human large intergenic noncoding RNAs reveals global properties and specific subclasses. Genes Dev. 25, 1915-1927 (2011).

65. Trapnell, C., Pachter, L. \& Salzberg, S. L. TopHat: discovering splice junctions with RNA-Seq. Bioinformatics 25, 1105-1111 (2009).

66. Trapnell, C. et al. Transcript assembly and quantification by RNA-Seq reveals unannotated transcripts and isoform switching during cell differentiation. Nat. Biotechnol. 28, 511-515 (2010).

67. Liao, Y., Smyth, G. K. \& Shi, W. featureCounts: an efficient general purpose program for assigning sequence reads to genomic features. Bioinformatics 30, 923-930 (2014).

68. White, N. M. et al. Transcriptome sequencing reveals altered long intergenic non-coding RNAs in lung cancer. Genome Biol. 15, 429 (2014).

69. Robinson, M. D., McCarthy, D. J. \& Smyth, G. K. edgeR: a bioconductor package for differential expression analysis of digital gene expression data. Bioinformatics 26, 139-140 (2010).

70. Stouffer, S. A. A study of attitudes. Sci. Am. 180, 11-15 (1949).

71. Jiang, H. \& Wong, W. H. SeqMap: mapping massive amount of oligonucleotides to the genome. Bioinformatics 24, 2395-2396 (2008).

72. Kavuri, S. M. et al. Cellular FLICE-inhibitory protein (cFLIP) isoforms block CD95- and TRAIL death receptor-induced gene induction irrespective of processing of caspase- 8 or cFLIP in the death-inducing signaling complex. $J$. Biol. Chem. 286, 16631-16646 (2011).

\section{Acknowledgements}

J.S.F. received funding from the Washington University School of Medicine Molecular Oncology Training Grant (T32CA113275). B.A.K., M.K.H., and J.G.G. received funding from the Washington University School of Medicine Surgical Oncology Basic Science and Translational Research Training Program (T32CA009621). R.C.F. and C.A.M. received funding from The Alvin J. Siteman Cancer Center Siteman Investment Program, The Foundation for Barnes-Jewish Hospital Cancer Frontier Fund, the National Cancer Institute Cancer Center Support Grant P30 CA091842, and the Barnard Trust. R.C.F. also received funding from the American Surgical Association Foundation Fellowship, American Cancer Society Institutional Review Grant, the Society of Surgical Oncology James Ewing Foundation Clinical Investigator Award, the Sidney Kimmel Translational Science Scholar Award, and the David Riebel Cancer Research Fund. Funding was also provided for C.A.M. by a Research Scholar Grant (130878-RSG-17-058-01-RMC) from the American Cancer Society and the NIH CTSA Grant \#UL1 TR002345. We would like to thank the Washington University's Genome Engineering and iPSC Center for help in the development of the CRISPR cell lines. We thank the Alvin J. Siteman Cancer Center at Washington University School of Medicine and Barnes-Jewish Hospital in St. Louis, MO., for the use of the Siteman Flow Cytometry, which provided flow cytometry service The Siteman Cancer Center is supported in part by an NCI Cancer Center Support Grant \#P30 CA091842. We would also like to thank the SAIC at Washington University for help with imaging mouse models and the Department of Medicine Pulmonary Morphology Core Division of Pulmonary and Critical Care Medicine for histology preparation. We also thank Jacqueline Payton, $\mathrm{MD}, \mathrm{PhD}$, for critically reviewing this manuscript.

\section{Author contributions}

J.S.F., R.C.F., and C.A.M. designed the project. J.S.F., H.X.D., N.M.W., A.C.L., R.C.F., and C.A.M. directed experimental studies. H.X.D., C.R.C., and A.E. performed sequencing data analysis. J.L. performed statistical analysis. J.S.F., N.M.W., M.S.S., B.A.K., E.B.R., G.G.L., J.G.G., M.K.H., and C.T. performed experimental studies. J.M. coordinated and processed biospecimens. J.S.F., H.X.D., N.M.W., C.R.C., R.C.F., and C.A.M. interpreted data. S.P.G., E.R.M., R.K.W., T.J.L., and R.C.F. provided project guidance. E.R.M., R.K.W., R.C.F., and C.A.M. provided financial support. J.S.F., H.X.D., N.M.W., and C.A.M. wrote and edited manuscript, which all authors reviewed.

\section{Competing interests}

The authors declare no competing interests.

\section{Additional information}

Supplementary information is available for this paper at https://doi.org/10.1038/s41467 020-15547-8.

Correspondence and requests for materials should be addressed to C.A.M.

Peer review information Nature Communications thanks Iain Tan and other, anonymous, reviewer(s) for their contribution to the peer review of this work. Peer reviewer reports are available.

Reprints and permission information is available at http://www.nature.com/reprints

Publisher's note Springer Nature remains neutral with regard to jurisdictional claims in published maps and institutional affiliations.

Open Access This article is licensed under a Creative Commons Attribution 4.0 International License, which permits use, sharing, adaptation, distribution and reproduction in any medium or format, as long as you give appropriate credit to the original author(s) and the source, provide a link to the Creative Commons license, and indicate if changes were made. The images or other third party material in this article are included in the article's Creative Commons license, unless indicated otherwise in a credit line to the material. If material is not included in the article's Creative Commons license and your intended use is not permitted by statutory regulation or exceeds the permitted use, you will need to obtain permission directly from the copyright holder. To view a copy of this license, visit http://creativecommons.org/ licenses/by/4.0/

(C) The Author(s) 2020 